\title{
MACROPHAGE-MEDIATED ANGIOGENIC ACTIVATION OF OUTGROWTH ENDOTHELIAL CELLS IN CO-CULTURE WITH PRIMARY OSTEOBLASTS
}

\author{
E. Dohle ${ }^{1, a, *}$, I. Bischoff ${ }^{1, a}$, T. Böse ${ }^{1}$, A. Marsano ${ }^{2}$, A. Banfi ${ }^{2}$, R. E. Unger ${ }^{1}$ and C. J. Kirkpatrick ${ }^{1}$ \\ ${ }^{1}$ Institute of Pathology, Johannes Gutenberg-University, Mainz, Germany \\ ${ }^{2}$ Departments of Surgery and Biomedicine, University Hospital Basel, Switzerland \\ ${ }^{\text {a }}$ Contributed equally
}

\begin{abstract}
The successful vascularisation of complex tissue engineered constructs for bone regeneration is still a major challenge in the field of tissue engineering. In this context, co-culture systems of endothelial cells and osteoblasts represent a promising approach to advance the formation of a stable vasculature as well as an excellent in vitro model to identify factors that positively influence bone healing processes, including angiogenesis. Under physiological conditions, the activation phase of angiogenesis is mainly induced by hypoxia or inflammation. Inflammatory cells such as macrophages secrete proinflammatory cytokines and proangiogenic growth factors, finally leading to the formation of new blood vessels. The aim of this study was to investigate if macrophages might positively influence the formation of microvessel-like structures via inflammatory mechanisms in a co-culture system consisting of human outgrowth endothelial cells (OECs) and primary osteoblasts. Treatment of co-cultures with macrophages (induced from THP-1) resulted in a higher number of microvessel-like structures formed by OECs compared to the co-culture. This change correlated with a significantly higher concentration of the proangiogenic VEGF in cell culture supernatants of triple-cultures and was accompanied by an increase in the expression of different proinflammatory cytokines, such as IL-6, IL-8 and TNF $\alpha$. In addition, the expression of E-selectin and ICAM-1, adhesion molecules which are strongly involved in the interaction between leukocytes and endothelial cells during the process of inflammation was also found to be higher in triple-cultures compared to the double co-cultures, documenting an ongoing proinflammatory stimulus. These results raise the possibility of actively using pro-inflammatory stimuli in a tissue engineering context to accelerate healing mechanisms.
\end{abstract}

Keywords: Tissue engineering; co-culture system; macrophages; angiogenic activation; inflammation; VEGF.

\author{
*Address for correspondence: \\ Dr E. Dohle \\ Institute of Pathology \\ Johannes Gutenberg-University \\ Langenbeckstr. 1 \\ 55101 Mainz \\ Germany
}

Telephone Number: +49-6131-17-6043

FAX Number +49-6131-17-5645

E-mail: dohle@uni-mainz.de
A successful clinical application of implanted cellconstructs for bone repair is strongly dependent on the formation of a functional vascular network supplying the construct with oxygen and nutrients. Several approaches have been proposed to improve the neovascularisation process of engineered tissues during the past decade, including the use of endothelial cells or endothelial progenitor cells (Grellier et al., 2009; Rivron et al., 2008; Rouwkema et al., 2006), strategies based on the natural interaction of the different cell types during the process of bone regeneration using co-culture systems (Fuchs et al., 2007; Kaigler et al., 2003) or additional treatment or coupling of tissue engineered constructs with proangiogenic growth factors or morphogens (Dohle et al., 2011; Dohle et al., 2010; Zisch et al., 2003).

In particular, the use of complex co-culture systems might be a beneficial tool to create prevascularised tissue engineered constructs for possible therapeutic applications (Finkenzeller et al., 2006; Mayer et al., 2005). Focus of the current study is on vascularisation using outgrowth endothelial cells, a subpopulation of endothelial progenitor cells, especially in the context of bone tissue engineering. The study is based on an in vitro co-culture model consisting of outgrowth endothelial cells (OECs) and primary osteoblasts (pOBs), which has the potential to permit further insight into underlying mechanisms of bone vascularisation and could also support the identification of factors stimulating the vascularisation process in therapeutic approaches. It has already been shown in a number of studies that OECs form lumen-containing, microvessel-like structures after 3 to 4 weeks of co-cultivation when seeded together with primary osteoblasts (Fuchs et al., 2007; Fuchs et al., 2009). The osteoblasts seem to provide proangiogenic matrix as well as proangiogenic cues that might lead to the angiogenic activation of OECs in long-term cocultures. For a better clinical application it would be useful to improve the beneficial effect of the co-culture on the angiogenic activation of OECs and to accelerate the formation of vessel-like structures observed in the co-culture. Therefore, additional treatment of co-cultures with vascular endothelial growth factor (VEGF) or the morphogen sonic hedgehog (Shh) result in pro-angiogenic activation as early as after $24 \mathrm{~h}$ (Dohle et al., 2011; Dohle et al., 2010). Nevertheless, treating co-cultures with growth factors or morphogens has several limitations and drawbacks, e.g. relatively short half-lives of growth factors or the induction of leaky and unstable vessels in response to VEGF treatment (Sundberg et al., 2002). 
Mimicking the natural responses of the human body to induce the formation of angiogenic structures might be another option. Under physiological conditions in the human body, angiogenesis, the formation of new blood vessels, is induced by hypoxia or inflammation. Inflammation starts when activated platelets of the fibrin clot release growth factors such as platelet derived growth factor (PDGF) and transforming growth factor beta (TGF $\beta$ ), thus attracting inflammatory cells such as neutrophils and monocytes (Tsaryk et al., 2007; Werner and Grose, 2003). Monocytes differentiate into macrophages at sites of inflammation and secrete proinflammatory cytokines including tumour necrosis factor alpha (TNF $\alpha$ ) and interleukins that amplify the inflammatory response (Werner and Grose, 2003). During the proliferative phase of wound healing, granulation tissue consisting of inflammatory cells, newly formed blood vessels and fibroblasts is formed. In order to recruit cells to sites of wound healing, macrophages secrete PDGF and TGF $\beta$ that induce the migration and proliferation of fibroblasts. Concurrently, macrophages and fibroblasts secrete proangiogenic factors such as VEGF and basic fibroblast growth factor (bFGF) which lead finally to new blood vessel formation (Adams and Alitalo, 2007; Diegelmann and Evans, 2004).

The aim of this study was to investigate whether macrophages might positively influence the formation of microvessel-like structures via inflammatory processes in the established co-culture system consisting of OECs and pOBs. Our working hypothesis was that the addition of the macrophage phenotype would indeed act as an inducer of neoangiogenesis in the human triple culture system. Therefore, co-cultures were treated with macrophageinduced THP-1 cell line (MФ) for 7 and $14 \mathrm{~d}$ before the cells were analysed in terms of inflammatory activation and angiogenesis activation using immunohistochemistry with image analysis, ELISA and quantitative real-time RT-PCR.

\section{Materials and Methods}

All cells were obtained from excess tissue and were used in accordance with the principle of informed consent and approved by the responsible Ethics Commission of the State of Rhineland-Palatinate, Germany.

\section{Endothelial cells}

Human OECs were isolated from peripheral blood buffy coats according to a previously published method (Fuchs et al., 2006). The cells were grown until confluence on fibronectin-coated $(10 \mu \mathrm{g} / \mathrm{mL}$, Milipore, Billerica, MA, USA) 24-well plates in EBM-2 medium (Lonza, Basel, Switzerland), supplemented with EGM-2 BulletKit (Lonza), $1 \%$ Penicillin/Streptomycin (Invitrogen, Carlsbad, CA, USA) and additional $4 \%$ foetal calf serum (FCS, Gibco, Carlsbad, CA, USA) so that the medium contained a final concentration of $7.5 \%$ serum. A total number of $5 \times 10^{6}$ cells per well were seeded on a 24-well plate. The cells were cultivated at $37^{\circ} \mathrm{C}$ in an atmosphere of $5 \% \mathrm{CO}_{2}$ and $95 \%$ air. After 3-4 weeks, colonies with a cobblestone-like morphology appeared. These late OECs with a typical mature endothelial marker profile and a high proliferation potential, were trypsinised and expanded on fibronectin-coated 24-well plates $(10 \mu \mathrm{g} / \mathrm{mL})$ over several passages using a splitting ratio of 1:2. Passage numbers of OECs used for this study ranged from passage 8 to passage 20 .

\section{Primary osteoblasts}

Human pOBs were isolated from cancellous bone fragments from healthy donors according to a previously published method (Hofmann et al., 2003) and cultivated in Dulbecco's Modified Eagle's Medium Nutrient Mixture F-12 (DMEM/F-12, Sigma-Aldrich, St. Louis, MO, USA), supplemented with $10 \%$ FCS (Gibco) $+1 \%$ Penicillin/ Streptomycin at $37{ }^{\circ} \mathrm{C}$ in an atmosphere of $95 \%$ air and $5 \% \mathrm{CO}_{2}$. Cells were passaged in a ratio of $1: 2$. For the present studies cells were used from several donors up to the third passage.

\section{Monocytic cell line THP-1}

The acute human monocytic leukemia cell line THP-1 was obtained from the ATCC (Manassas, VA, USA). Cells were grown in suspension at $1.2 \times 10^{5}$ cells $/ \mathrm{mL}$ in RPMI 1640 medium containing $10 \%$ FCS and $1 \%$ Penicillin/ Streptomycin and maintained at $37^{\circ} \mathrm{C}$ in an atmosphere of $95 \%$ air and $5 \% \mathrm{CO}_{2}$. To fully differentiate the monocytes to macrophages (MФ), $5 \times 10^{5}$ THP-1 monocytic cells were seeded on a fibronectin-coated ( $5 \mu \mathrm{g} / \mathrm{mL}$, Milipore) 6 -wellplate on a growth area of $9.6 \mathrm{~cm}^{2}$ in $3 \mathrm{~mL}$ RPMI medium (Gibco) containing 8 nM PMA (phorbol-12-myristate-13acetate) (Sigma-Aldrich) for $4 \mathrm{~d}$. Unattached cells were removed by washing with PBS (Dulbecco's Phosphate Buffered Saline, Gibco).

\section{BMSC transduction}

Bone marrow-derived mesenchymal stromal cells (BMSC) were isolated as previously described (Horn et al., 2008). Briefly, fresh marrow was plated at a density of $10^{5}$ nucleated cells $/ \mathrm{cm}^{2}$ and cultured in high glucose DMEM (Gibco) supplemented with $10 \%$ FBS and 5 ng/mL FGF2 (BD Biosciences, Basel, Switzerland). BMSC were transduced in $\mathrm{P} 0$ before reaching confluence with retroviral vectors, carrying the gene for the truncated extracellular domain of mouse VEGF Receptor-2 (sFlk1) fused to the IgG2 $\alpha$ Fc domain (Kuhnert et al., 2008), linked through an Internal Ribosomal Entry Site (IRES) to a truncated version of mouse CD8a as a cell surface marker, according to a high-efficiency protocol as previously described (Helmrich et al., 2012). The percentage of transduced cells was quantified by flow cytometry with a FACSCalibur flow cytometer (BD Biosciences, San Jose, CA, USA), after staining with an allophycocyanin-conjugated anti-mouse CD8a antibody (clone 53-6.7; BD Biosciences, USA), and analysed with FlowJo software (Tree Star, Ashland, OR, USA). Transduced BMSC were replated at a density of $10^{4}$ cells $/ \mathrm{cm}^{2}$ in $60-\mathrm{mm}$ dishes, and, after incubation with $1 \mathrm{~mL}$ of fresh culture medium for $4 \mathrm{~h}$, supernatants 
A

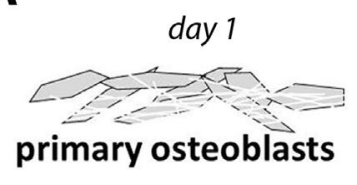

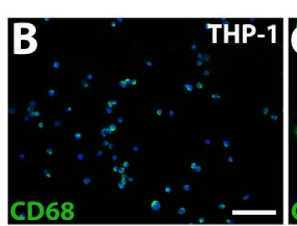

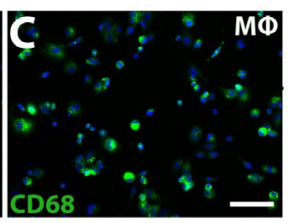

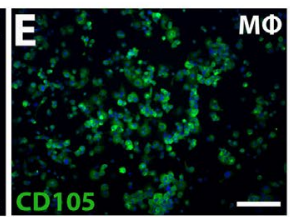

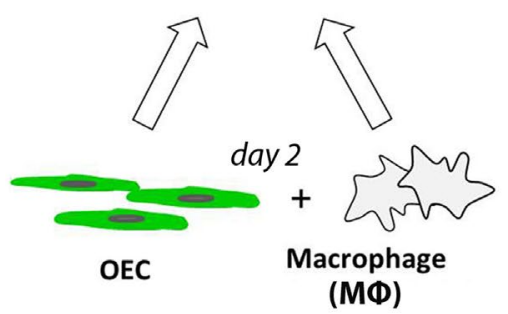

H

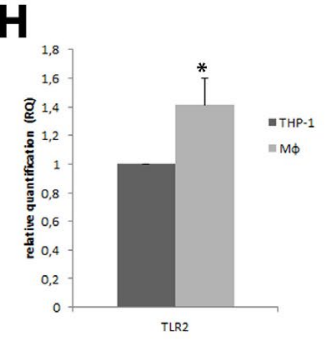

$\mathbf{L}$

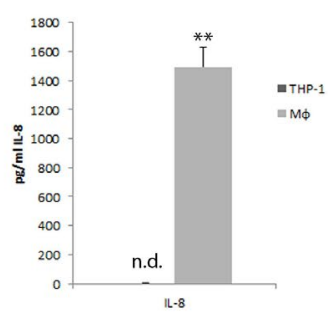

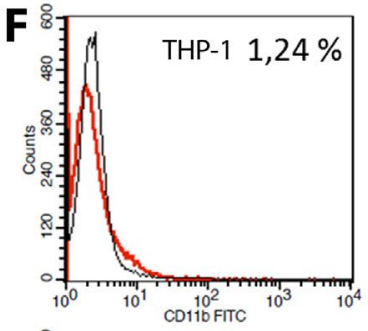

I

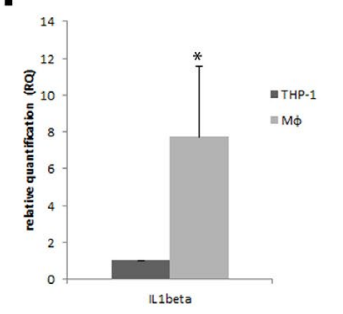

M

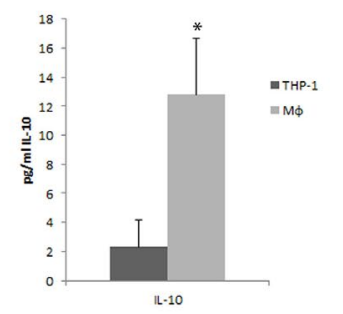

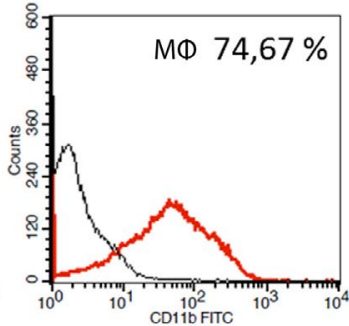

G

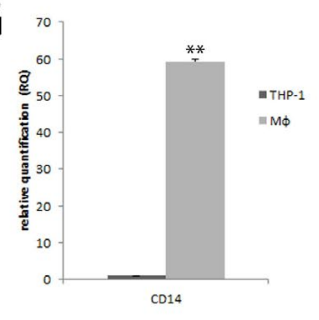

K
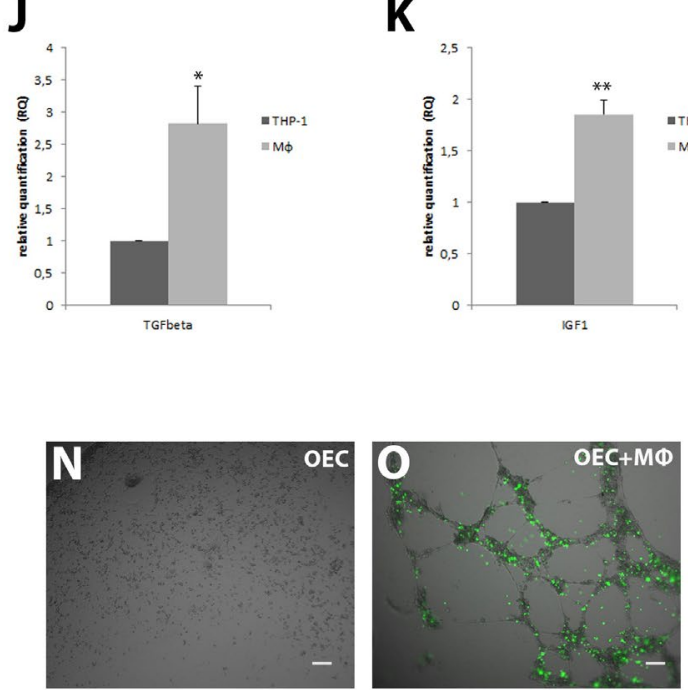

Fig. 1. (A) Culture conditions for triple-culture. Primary human osteoblasts were seeded on day 1 at a ratio of 300,000 cells per well of a 24-well plate. On day 2, 200,000 OEC and 30,000 macrophage-induced THP-1 (MФ) were added. Coand triple-cultures were cultivated for 7 and $14 \mathrm{~d}$. (B,D) THP-1 monocytic cells stained negatively for the macrophage marker CD68 and CD105 $(n=3$; scale bar $100 \mu \mathrm{m})$. (C,E) When cells were treated with $8 \mathrm{nM}$ PMA for $4 \mathrm{~d}$, they exhibited the typical macrophage phenotype and stained positively for CD68 and CD105 ( $n=3$; scale bar $100 \mu \mathrm{m}$ ). (F) Flow cytometry. Representative histogram plot for the pan-macrophage marker CD11b (red line) and the isotypic control (black line) in THP-1 cells and PMA-treated cells $(\mathrm{M} \Phi)(n=3)$. (G) Relative gene expression of CD14 in THP-1 and MФ evaluated using quantitative real time PCR $(n=4)$. $(\mathbf{H})$ Relative gene expression of TLR2 in THP-1

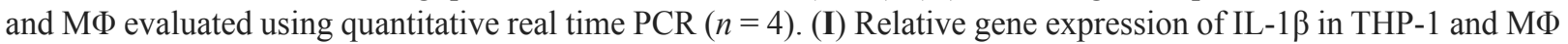
evaluated using quantitative real time PCR $(n=4)$. (J) Relative gene expression of TGF $\beta$ in THP-1 and MФ evaluated using quantitative real time PCR $(n=4)$. (K) Relative gene expression of IGF1 in THP-1 and MФ evaluated using quantitative real time PCR $(n=4)$. $(\mathbf{L})$ Protein release of IL-8 in THP-1 and MФ evaluated using ELISA $(n=4$; n.d. $=$ not detectable). (M) Protein release of IL-10 in THP-1 and MФ evaluated using ELISA $(n=4)$. (N) OEC mono-culture as well as (O) OEC in double co-culture with macrophage-induced THP-1 (Calcein-AM labelled; green fluorescence) seeded on Matrigel basement membrane matrix and cultivated for $14 \mathrm{~h}(n=4)$. Scale bars $200 \mu \mathrm{m}$.

were collected, filtered through $0.45 \mu \mathrm{m}$ syringe filters and frozen at $-80{ }^{\circ} \mathrm{C}$ until use. Supernatants collected by nontransduced (naïve) BMSC were used as controls.

\section{Conditions for co- and triple-cultures}

OECs and pOBs were seeded in a ratio of 1:1.5 in EBM2 medium as previously published (Fuchs et al., 2007). Double co-cultures of outgrowth endothelial cells and primary osteoblasts were grown on fibronectin-coated
(10 $\mu \mathrm{g} / \mathrm{mL}$ Millipore) Thermanox coverslips (12 $\mathrm{mm}$ in diameter, Nunc, Roskilde, Denmark) in 24-well plates $\left(1,766 \mathrm{~cm}^{2}\right)$, always seeding primary osteoblasts first (300,000 cells/well). After one day OECs were added (200,000 cells/well) together with THP-1-derived macrophages $(\mathrm{M} \Phi)$ at 30,000 cells per well (Fig. 1A). For immunofluorescence staining, triple- and co-cultures were seeded in fibronectin-coated Petri dishes $(35 \mathrm{~mm})$ to avoid autofluorescence. The triple-cultures as well as 
control co-cultures were cultivated in EBM-2 medium supplemented with EGM-2 BulletKit, 1 \% Penicillin/ Streptomycin and additional $4 \%$ foetal calf serum for 7 and $14 \mathrm{~d}$ at $37{ }^{\circ} \mathrm{C}$ in an atmosphere of $5 \% \mathrm{CO}_{2}$ and $95 \%$ air. In control experiments, co-cultures of pOBs and OECs were additionally treated with conditioned medium derived from activated THP-1 (MФ) for 7 and $14 \mathrm{~d}$ under the same conditions. For all co- and triple-culture experiments, at least 3 donors were used. The analysed donor number per experiment is individually addressed in the results section and ranges from $n=3$ to $n=7$.

\section{Enzyme-linked immunosorbent assay (ELISA)}

Culture supernatants were collected from co-cultures consisting of pOBs and OECs and triple-cultures composed of pOBs, OECs and THP-1-derived macrophages (MФ) after 7 and $14 \mathrm{~d}$ of cultivation as well as from the appropriate mono-cultures and differently combined double co-cultures (pOBs $+\mathrm{M} \Phi$ and $\mathrm{OECs}+\mathrm{M} \Phi$ ) serving as controls. The concentration of VEGF was measured using DuoSet $^{\circledR}$ ELISA Development Systems (R\&D Systems, Minneapolis, MN, USA) according to the manufacturer's protocol. Samples were measured in triplicate using a microplate reader (GENios plus, TECAN, Crailsheim, Germany) at a wave length of $450 \mathrm{~nm}$. Soluble Flk1 was quantified in BMSC conditioned supernatants using a mouse-specific Quantikine immunoassay ELISA kit (R\&D Systems, Abingdon, UK) according to the manufacturer's instructions.

\section{Quantitative real-time reverse transcriptase- polymerase chain reaction \\ (Quantitative real-time PCR)}

RNA was isolated using a Qiagen RNEasy Micro Kit (Qiagen, Hilden, Germany). To obtain a well-defined quantity of cDNA, $1 \mu \mathrm{g}$ of extracted RNA was used for reverse transcription by the Omniscript RT kit (Qiagen) according to the manufacturer's protocol. The following primers were used for this study: CD14, interleukin-1ß (IL-1ß), insulin-like growth factor 1 (IGF-1), TGFß, VEGF-A, E-selectin, intercellular adhesion molecule-1 (ICAM-1), interleukin-6 (IL-6), interleukin-8 (IL$8)$, TNF- $\alpha$, osteocalcin, osteopontin and runt-related transcription factor 2 (RUNX2). 60S ribosomal protein L13A (RPL13A) was used as endogenous control All primers were commercially obtained from Microsynth (Balgach, Switzerland). $4 \mathrm{ng}$ of cDNA was used for one reaction. Quantitative real-time PCR (7300 Real Time PCR System; Applied Biosystems, Applera Deutschland GmbH, Darmstadt, Germany) was performed in triplicate with the following cycler program: $95{ }^{\circ} \mathrm{C}$ for $10 \mathrm{~min}, 95{ }^{\circ} \mathrm{C}$ for $15 \mathrm{~s}$ and $60^{\circ} \mathrm{C}$ for $1 \mathrm{~min}, 40$ cycles. To specify the length of the DNA fragments, a dissociation stage was added to the protocol: $95{ }^{\circ} \mathrm{C}$ for $15 \mathrm{~s}, 60^{\circ} \mathrm{C}$ for $1 \mathrm{~min}, 95^{\circ} \mathrm{C}$ for $15 \mathrm{~s}$ and $60{ }^{\circ} \mathrm{C}$ for $15 \mathrm{~s}$. Relative gene expression was determined using the $\Delta \Delta \mathrm{Ct}$ method. Gene expression was compared by setting control cultures to 1 (reference value) as indicated in the relevant figures. For gene expression studies of the triple-culture, the appropriate mono-cultures and differently combined double co-cultures (pOBs $+\mathrm{M} \Phi$ and $\mathrm{OECs}+\mathrm{M} \Phi)$ were also analysed for the specific marker expression, serving as controls.

\section{Immunofluorescence staining}

To differentiate between non-activated and macrophageactivated THP-1, cells were stained immunofluorescently for CD68 diluted 1:100 (mouse; Santa Cruz Biotechnology, Santa Cruz, CA, USA) and CD105 (1:25; DAKO, Hamburg, Germany) before and after treatment with PMA. Co-cultures were stained for the specific endothelial marker CD31 (1:50, goat; Santa Cruz Biotechnology) after 7 and $14 \mathrm{~d}$ of cultivation. Triple-cultures were additionally stained for the macrophage-specific marker CD68 (1:100, mouse). The cell nuclei of all cultures were stained by Hoechst dye. After fixation with $3.7 \%$ paraformaldehyde (Merck, Darmstadt, Germany), the cells were permeabilised with $0.5 \%$ Triton X/PBS and washed three times with PBS. The co-cultures were incubated with the primary antibody diluted in a $1 \%$ bovine serum albumin/PBS solution for $60 \mathrm{~min}$ at room temperature. After washing three times with PBS, the cells were incubated with the secondary anti-goat antibodies Alexa 488 (donkey; Molecular Probes, MoBiTec, Göttingen, Germany), and Alexa 546 (rabbit; Molecular Probes) diluted 1:1000 in a $1 \%$ bovine serum albumin/PBS solution for $60 \mathrm{~min}$ at room temperature, protected from light. The cells were mounted with Fluoroshield (ImmunoBioScience Corp., Mukilteo, WA, USA) and examined using a confocal laser scanning microscope (LeicaTCS-NT, Leica Microsystems, Wetzlar, Germany) or a conventional fluorescence microsope (Biozero, Keyence, Neu-Isenburg, Germany).

\section{Quantification of mineralisation using alizarin red}

To quantify the mineralisation in co- and triple-cultures, an Osteogenesis Quantitation Kit was used according to the manufacturer's protocol (Chemicon International, Hofheim, Germany). Co- and triple-cultures were cultured in EBM-2 medium supplemented with EGM-2 BulletKit, $1 \%$ Penicillin/Streptomycin and additional $4 \%$ foetal calf serum for 7 and $14 \mathrm{~d}$ at $37{ }^{\circ} \mathrm{C}$ in an atmosphere of $5 \%$ $\mathrm{CO}_{2}$ and $95 \%$ air. Quantitative analysis was performed by extraction of the stain. Alizarin red concentration was measured on a transparent 96-well plate in triplicates at $\mathrm{OD}_{405}$ using a microplate reader (GENios plus). Alizarin red concentration was defined as $\mu \mathrm{M}$ Alizarin $\mathrm{red} / \mathrm{mg}$ protein. ABCA (bicinchoninic acid) protein Assay Reagent Kit (Pierce, Thermo Fischer, Bonn, Germany) was used to determine the protein concentration according to the manufacturer's instructions.

\section{Image quantification}

The microscope images were analysed semi-automatically using the image processing software Fiji. From each image, the intensity of the fluorescent background was subtracted using a rolling ball radius determined from the radius of the largest object that was not part of the background (Sternberg, 1983). Vascular structures were extracted from the remaining images by automatic thresholding. The resulting binary images were manually corrected as overlays on the original microscope pictures. 
From the black and white pixels of the binaries the area of angiogenic structures was measured. The mean vessel length and the mean number of branches were determined after skeletonisation of the vascular networks. Similarly, the number of branch points was calculated by defining the total number of pixels with more than two neighbours. All calculations were performed using MS Excel (Microsoft Office, Microsoft, München, Germany).

\section{Characterisation of monocyte-derived macrophages using flow cytometry}

After $4 \mathrm{~d}$ of PMA treatment, culture cells were harvested by trypsinisation $(0.25 \%$ Trypsin-EDTA, Gibco/Life Technologies, Darmstadt, Germany), washed with PBS and centrifuged at $400 \mathrm{~g}$ for $5 \mathrm{~min}$. For appropriate blocking, the resulting cell pellet was resuspended in PBS containing $1 \%$ BSA (Sigma-Aldrich). Subsequently, $3 \times 10^{5}$ cells per sample were stained for $15 \mathrm{~min}$ at $4{ }^{\circ} \mathrm{C}$ using CD11b-FITC (11-0118-42, eBioscience, San Diego, CA, USA) and IgG1-FITC (11-4714, eBioscience). Cells were washed with $3 \mathrm{~mL}$ fresh PBS and centrifuged at $400 \mathrm{~g}$ for $5 \mathrm{~min}$. After discarding the supernatant the remaining cell pellet was resuspended in $500 \mu \mathrm{L}$ fresh PBS. The stained cells were immediately analysed for the expression of CD11b using a FACSCalibur flow cytometer combined with the CellQuestPro Software (BD Bioscience, USA). The analyses were performed as duplicates of 50,000 counted events.

\section{Matrigel angiogenesis assay \\ Matrigel ${ }^{\circledR}$ Basement Membrane Matrix (Becton Dickinson Labware, Bedford, UK) was thawed on ice at $4{ }^{\circ} \mathrm{C}$ overnight and diluted 1:2 in cold EBM-2 with supplements from the kit $/ 5 \% \mathrm{FCS}$ and $1 \% \mathrm{P} / \mathrm{S} .50 \mu \mathrm{L}$ of this dilution was added to each well of a 96-well plate and incubated for $30 \mathrm{~min}$ at $37^{\circ} \mathrm{C}$ to allow the polymerisation of the Matrigel. 50,000 OECs per well of a 96-well plate were resuspended in $200 \mu \mathrm{L}$ of EBM-2 and gently pipetted on top of the solidified Matrigel ${ }^{\circledR}$ Basement Membrane Matrix. 7,000 macrophage-induced THP-1 (MФ) were incubated with the live cell stain Calcein AM (1:1000; Invitrogen) for $5 \mathrm{~min}$ at $37^{\circ} \mathrm{C}$ before being added to the OECs. $14 \mathrm{~h}$ later, cells were imaged using a fluorescence microscope (Biozero).}

\section{Statistical analysis}

All data are presented as mean values \pm standard error of the mean. Statistical significance was evaluated using the paired Student's $t$-test and was performed with Excel. This test was chosen to compare triple-cultures and double cocultures from the same individuals. Statistical significance was assessed by $\underline{p}$-value $* p<0.05$ or $* * p<0.03$, respectively.

\section{Results}

\section{Angiogenic activation of OECs in the co-culture system in response to $\mathrm{M \Phi}$ treatment}

The THP-1 cell line resembles primary monocytes in morphology as well as in differentiation capacity and marker profile (Fig. 1B-M). Non-activated THP-1 cells stained negatively for the macrophage markers CD68 and CD105 (Fig. 1B,D, $n=3$ ). When THP-1 cells were treated with PMA, they started to adhere and change their monocytic phenotype to a macrophage phenotype $(\mathrm{M} \Phi)$, indicated by a positive staining for CD68 and CD105 (Fig. $1 \mathrm{C}, \mathrm{E}, n=3$ ). The ratio of $\mathrm{CD} 11 \mathrm{~b}$ positive cells, a well-known pan-macrophage marker, increased from $1.24 \%$ to $74.67 \%$ when THP-1 cells were treated with PMA (Fig. 1F, $n=3$ ). In addition, the expression of CD14 and toll-like receptor 2 (TLR2) were also significantly upregulated in MФ compared to THP-1, documenting an ongoing differentiation triggered through PMA treatment (Fig. $1 \mathrm{G}, \mathrm{H}, n=4$ ). Different macrophage markers such as IL-1ß, TGFß and IGF1 were also found to be upregulated when the THP-1 cell line was treated with PMA (Fig. $1 \mathrm{I}, \mathrm{J}, \mathrm{K}, n=4)$. Furthermore, the activation of THP-1 into $\mathrm{M} \Phi$ was accompanied by a significantly higher release of the macrophage-specific cytokines IL-8 and IL-10 (Fig. 1L,M, $n=4)$.

It has already been documented in previous studies that co-cultivation of pOBs and OECs leads to the formation of angiogenic structures by OECs in long-term cultures and under different culture conditions, dependent on growth factor treatment. This makes the established co-culture system a highly relevant tool to study important processes essential for bone repair, especially inflammation-induced angiogenic activation. In a first step, the effects of macrophage-induced THP-1 on the angiogenic activation of OEC mono-culture seeded on Matrige ${ }^{\circledR}$ basement membrane matrix was analysed (Fig. 1N,O). This angiogenesis assay clearly demonstrates the arrangement of OECs into tube-like structures in response to addition of THP-1, which clearly accumulate at sites of angiogenic structures formed by OECs (Fig. 1O). In contrast, OEC in mono-culture do not form angiogenic structures without the addition of $\mathrm{M} \Phi($ Fig. $1 \mathrm{~N})$. Cells retain their cobblestone-like morphology, failing to organise into tube-like structures in the growth factor-reduced Matrigel without any angiogenic stimulus. Based on this first result, macrophage-induced THP-1 (MФ) were added to the cocultures of pOBs and OECs, and were triple-cultured for 7 and $14 \mathrm{~d}$ and analysed in terms of the angiogenic activation of OECs in the triple-culture (Fig. 2A-E). Interestingly, treatment of co-cultures consisting of pOBs and OECs with macrophage-induced THP-1 (MФ) results in an increase of the formation of microvessel-like structures after both time points of triple-cultivation, as indicated by immunofluorescence staining for the endothelial marker CD31 (Fig. 2B,C,E, $n=7$ ). Furthermore, CD68-positive cells, representing macrophage-induced THP-1, can be detected in microvessel-adjacent areas (Fig. 2C',E', arrows). In contrast, control co-cultures (Fig. 2A,D) stained for CD31 exhibited a lower amount of angiogenic structures after 7 and $14 \mathrm{~d}$ of co-cultivation. Although, the formation of angiogenic structures has already started in control-cultures (arrows), there were still a number of cells organised in cell colonies detectable after both cultivation time points, indicating a later onset of angiogenic activation in the absence of macrophages (Fig. 2A,D). Subsequently, vascular area as well as the mean for branch points, 

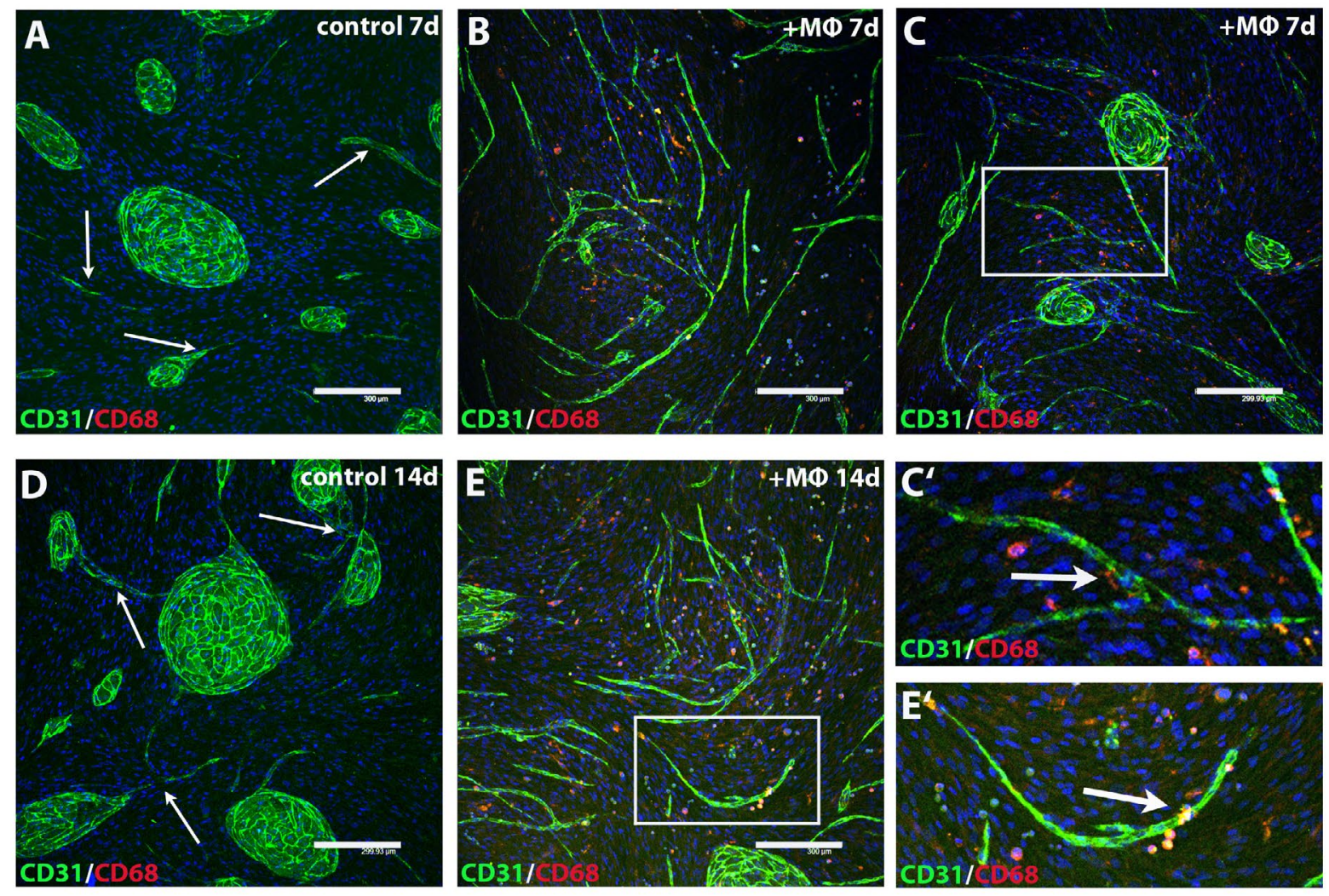

\section{F}
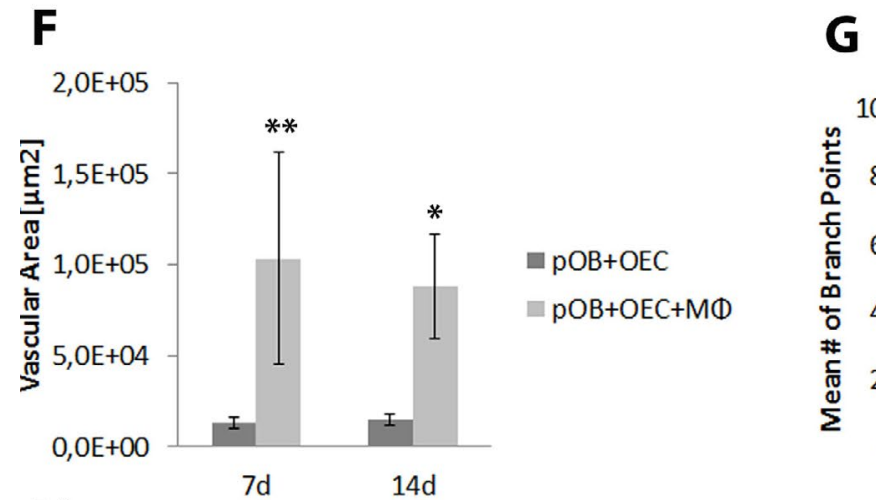

\section{H}
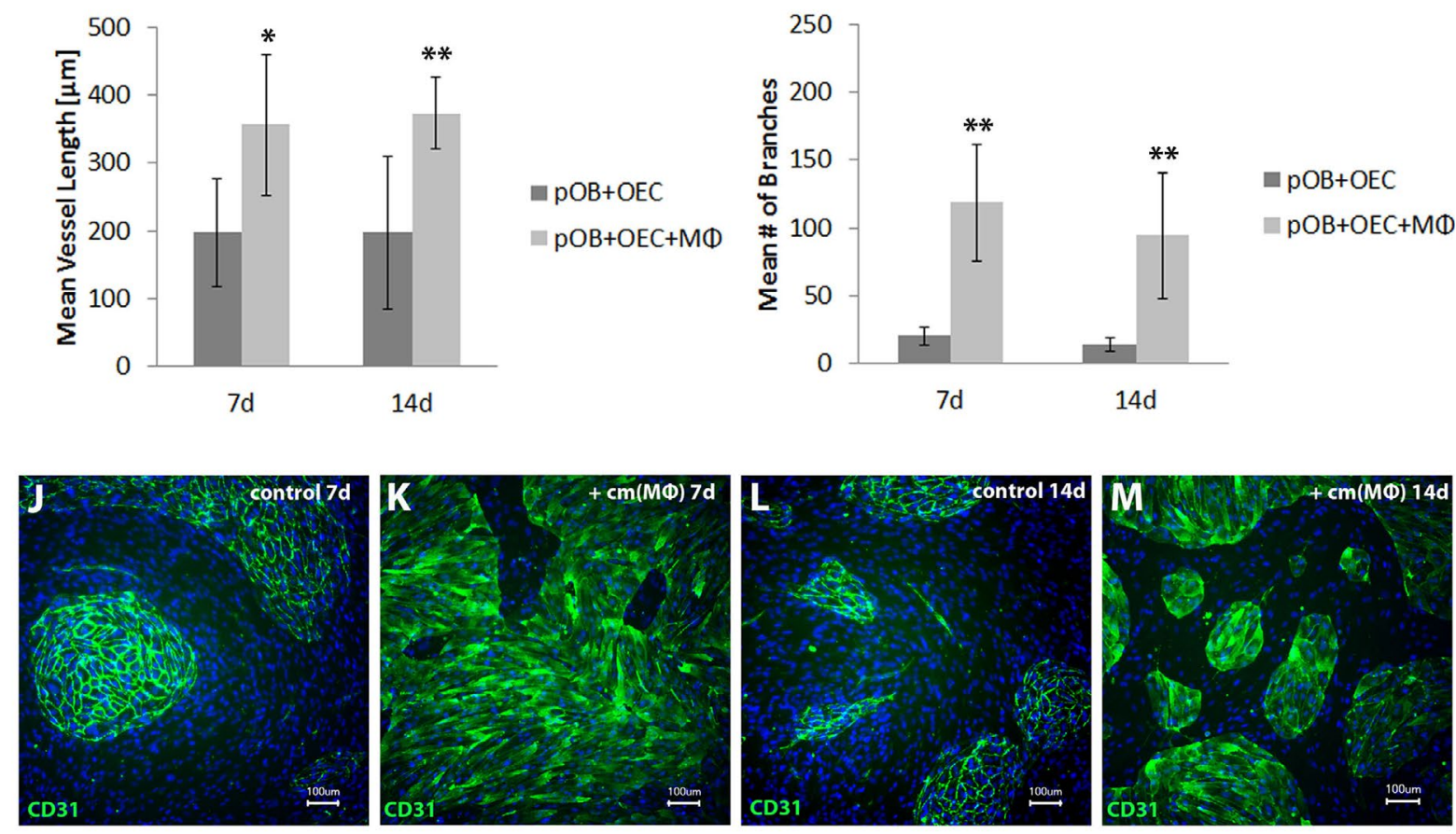
Fig. 2. (Figure on previous page - 154) Angiogenic activation of OECs in the triple-culture. (A-E) Immunofluorescence staining of co- and triple-cultures. Double co-cultures consisting of pOB and OEC as well as triple-cultures of pOBs, OECs and macrophage-induced THP-1 (MФ) were cultivated for $7 \mathrm{~d}(\mathbf{A}, \mathbf{B}, \mathbf{C})$ and $14 \mathrm{~d}(\mathbf{D}, \mathbf{E})$ and stained for the endothelial marker CD31 (green) and for the macrophage marker CD68 (red). The formation of angiogenic structures can be detected in $7 \mathrm{~d}$ triple-cultures $(\mathbf{B}, \mathbf{C})$ as well as in $14 \mathrm{~d}$ of triple-cultivation $(\mathbf{E})$ as seen by positive staining of microvessel-like structures for CD31. By comparison, double co-cultures of pOB and OEC $(\mathbf{A}, \mathbf{D})(n=7)$ show only a small degree of sprout formation at $7 \mathrm{~d}(\mathbf{A})$ and at $14 \mathrm{~d}(\mathbf{D})$ much less than in the triple culture (E). Higher magnification shows aggregation of macrophage induced THP-1 in areas of vessel-like structures formed by OEC (arrows, C',E'). Scale bars $150 \mu \mathrm{m}$. (F-I) Using image analysis angiogenic structures were quantified by comparing vascular area $(\mathbf{F})$, the mean of branch points $(\mathbf{G})$, the mean of vessel length $(\mathbf{H})$, as well as the mean of branches $(\mathbf{I})$ of macrophage-treated cultures and co-cultures after 7 and after $14 \mathrm{~d}$ of THP-1 exposure (MФ). $(n=3)$. Statistical significance is marked as $* p<0.05$ and $* * p<0.03$. (J-M) In control experiments, co-cultures consisting of pOBs and OECs were treated with conditioned medium derived from MФ cell cultures ( $\mathrm{cm} \mathrm{M \Phi )} \mathrm{and} \mathrm{stained} \mathrm{immunofluorescently}$ for the endothelial maker CD31. The formation of angiogenic structures could not be induced using $\mathrm{cm} \mathrm{M \Phi} \mathrm{(K,M),}$ neither after $7 \mathrm{~d}$ nor $14 \mathrm{~d}$ of treatment and compared to the control cultures $(\mathbf{J}, \mathbf{L})(n=3)$. Scale bars $100 \mu \mathrm{m}$.

vessel length and branches in co- and triple-cultures were quantified for both cultivation time points and confirmed the results of fluorescence microscopy (Fig. 2F-I, $n=3$ ). In general, a significantly higher number of microvessellike structures can be observed in triple-cultures of pOBs, OECs and МФ compared to the double co-cultures without MФ. Furthermore, treating co-cultures with conditioned medium from macrophage-induced THP-1 (MФ) failed to induce the formation of angiogenic structures, both after $7 \mathrm{~d}$ of cultivation and after $14 \mathrm{~d}$ (Fig. $2 \mathrm{~J}-\mathrm{M}, n=3$ ).

\section{МФ treatment of co-cultures consisting of pOBs and OECs induces an inflammatory response}

In order to analyse if treatment of co-cultures of pOBs and OECs with THP-1 (MФ) results in an inflammatory response that might lead to the angiogenic activation of OECs in the co-culture system, the expression of different proinflammatory cytokines, such as IL-6, IL-8 and TNF $\alpha$, was evaluated on the mRNA level using quantitative realtime RT-PCR (Fig. 3A,C,E, $n=7$ ). IL-6 and TNF $\alpha$ are the major proinflammatory cytokines that are responsible for the early inflammatory response, with IL-8 essentially involved in this acute inflammation, by inducing trafficking and activation of neutrophils along the vessel wall. In response to treatment of co-cultures with $M \Phi$, the expression of IL-6, IL-8 and TNF $\alpha$ was upregulated after $7 \mathrm{~d}$ as well as after $14 \mathrm{~d}$ of triple-cultivation. Statistical significance was established for IL-6 and IL-8 expression after $14 \mathrm{~d}$ of triple-culturing compared to the mRNA expression of these proteins in the double co-culture (Fig. $3 \mathrm{~A}, \mathrm{C}, * p<0.05)$. In order to obtain a first conception about the potential origin of the analysed factors in the triple-culture system, additional real time RT-PCR studies were performed to evaluate the gene expression level of IL-6, IL- 8 and TNF $\alpha$ in the appropriate mono-cultures as well as in differently combined double co-cultures $(\mathrm{OEC}+\mathrm{M} \Phi$ and $\mathrm{pOB}+\mathrm{M} \Phi)$ (Fig. 3B,D,F, $n=3$ ). Although no statistical significance could be determined for the 7 day time point, in contrast to the clear upregulation of all tested proinflammatory cytokines in the triple-culture, there is quite a low expression of these factors in the appropriate mono-cultures after $7 \mathrm{~d}$ of cultivation (Fig. 3B,D,F). After
$14 \mathrm{~d}$ of cultivation, IL- 6 , IL- 8 and TNF $\alpha$ expression is increased in pOB- and OEC-mono-cultures as well as in $\mathrm{OEC}+\mathrm{M} \Phi$ co-cultures, but not in co-cultures consisting of pOB $+\mathrm{M} \Phi$ and $\mathrm{M} \Phi$ mono-cultures (Fig. 3B,D,F, ${ }^{*} p<0.05$ ).

In addition, the expression of different adhesion molecules that are strongly involved in the interaction of endothelial cells and leucocytes, in particular macrophages, during the process of inflammation was also assessed on the mRNA level using quantitative real time RT-PCR (Fig. $4 \mathrm{~A}, \mathrm{C}, n=7$ ). During inflammation, adhesion of endothelial cells with macrophages starts with leucocyte rolling, which is mainly mediated through E-selectin, followed by more stable adhesion mediated through ICAM-1. A clear upregulating trend in the gene expression of E-selectin (Fig. 4C) and ICAM-1 (Fig. 4A) was observed when co-cultures were treated with macrophage-induced THP-1 (MФ) compared to the double co-cultures alone, documenting an ongoing proinflammatory stimulus in the triple-culture consisting of pOBs, OECs and MФ. Statistical significance was reached for the expression of ICAM-1 after $7 \mathrm{~d}$ of treatment and for E-selectin in response to $7 \mathrm{~d}$ and $14 \mathrm{~d}$ of macrophage treatment (Fig. 4A,C, $n=7 * p<0.05$ ). Evaluating the gene expression level of ICAM-1 and E-selectin also in the appropriate mono-cultures as well as in differently combined double co-cultures (OEC+M $\Phi$ and $\mathrm{pOB}+\mathrm{M} \Phi)$ revealed a high expression of ICAM-1 in OEC mono-cultures as well as in co-cultures consisting of OEC $+\mathrm{M} \Phi$ after both cultivation time points (Fig. 4B, $n=3$, $* p<0.05$ and $* * p<0.03)$. The latter data were expected, as these cell adhesion molecules are important markers of the endothelium. In general, E-selectin expression seems to be higher in $\mathrm{OEC}$ or $\mathrm{OEC}+\mathrm{M} \Phi$ co-cultures compared to $\mathrm{pOB}$ and co-cultures of $\mathrm{pOBs}+\mathrm{M} \Phi$ after $14 \mathrm{~d}$ of cultivation. Nevertheless, statistical significance was not achieved (Fig. $4 \mathrm{D}, n=3)$.

\section{Effects of $М \Phi$ on the expression of the proangiogenic factor VEGF in co-cultures of pOBs and OECs}

Following the process of acute inflammation, VEGF, a major proangiogenic factor, is secreted by macrophages and fibroblastic cells, which finally leads to increased vessel permeability, endothelial migration and new blood 


\section{A}

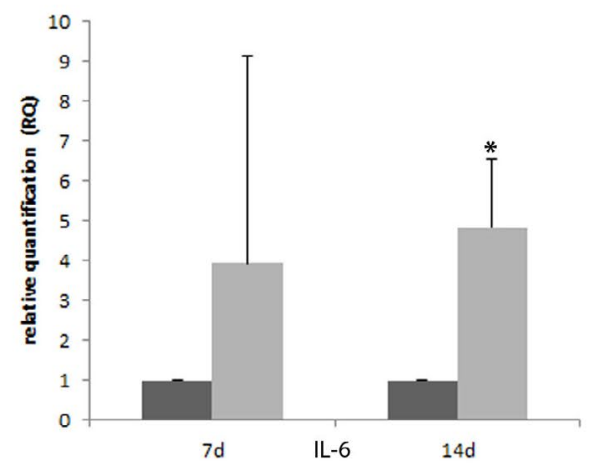

C

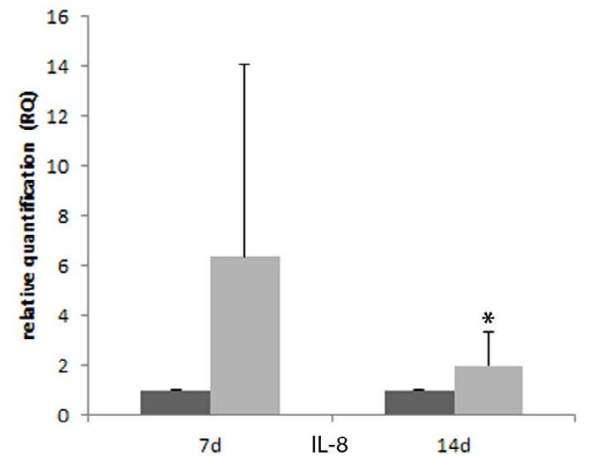

\section{E}

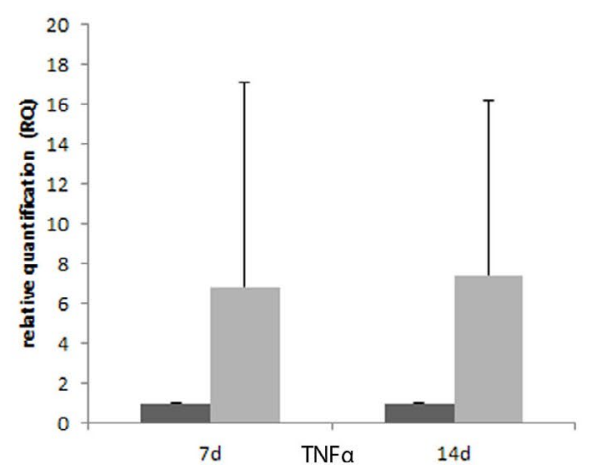

B

DOB+OEC

$\square \mathrm{DOB}+\mathrm{OEC}+\mathrm{M} \phi$

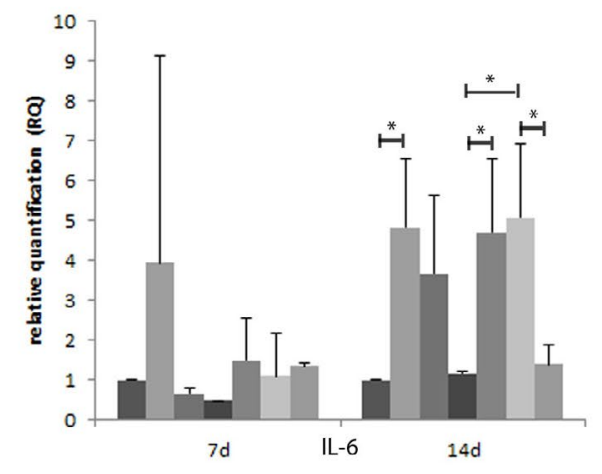

MOB+OEC

DOB+OEC+M $\phi$

$\square$ ров

- $\mathrm{pOB}+\mathrm{M} \phi$

$\because \mathrm{OEC}$

$=\mathrm{OEC}+\mathrm{M} \phi$

- $\mathrm{M} \phi$
- $\mathrm{DOB}+\mathrm{OEC}$

DOB+OEC+M $\phi$

- $\mathrm{POB}+\mathrm{OEC}$

$\mathrm{DOB}+\mathrm{OEC}+\mathrm{M} \phi$
D

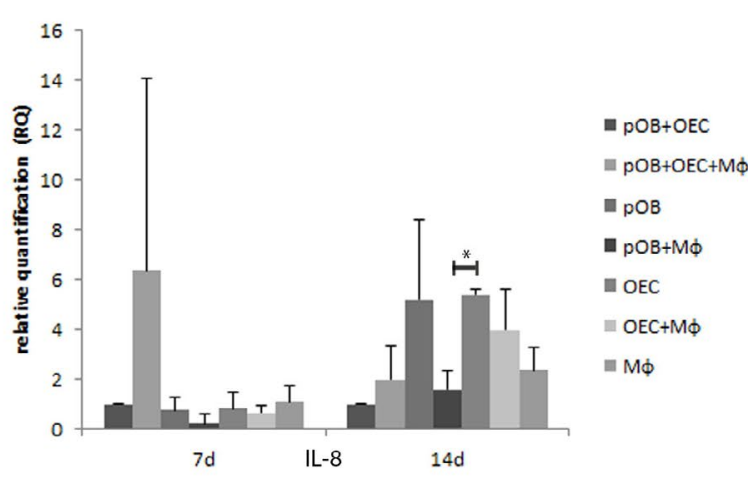

F

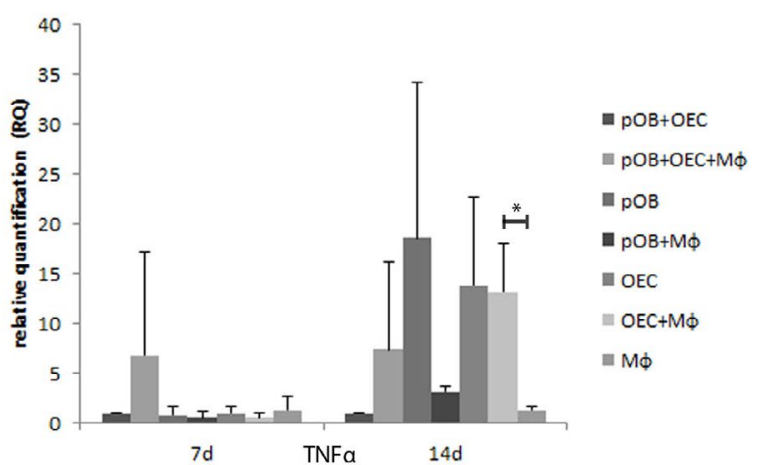

Fig. 3. Proinflammatory effect of macrophage treatment on the co-culture consisting of pOBs and OECs. The inflammatory cytokines IL-6 (A), IL-8 $(\mathbf{C})$ and TNF $\alpha(\mathbf{E})$ are upregulated in the co-culture system in response to treatment with macrophage-induced THP-1 (MФ) after $7 \mathrm{~d}$ as well as $14 \mathrm{~d}$ of triple-culturing evaluated using quantitative real time PCR analysis. Statistical significance can be detected for the macrophage-induced upregulation of IL-6 and IL-8 after $14 \mathrm{~d}$ of macrophage exposure (A,C, $* p<0.05)$. $(n=7)$. (B,D,F) In order to gain insight into the potential origin of IL-6, IL- 8 and TNF $\alpha$ in the triple-culture, the expression of these molecules was also assessed in mono-cultures as well as in $\mathrm{OEC}+\mathrm{M} \Phi$ and $\mathrm{pOB}+\mathrm{M} \Phi$ co-cultures. Relative gene expression was determined using the $\Delta \Delta \mathrm{Ct}$ method and gene expression was compared by setting control co-cultures to 1 ( $\mathrm{pOB}+\mathrm{OEC}$ cultivated for $7 \mathrm{~d}$ and $\mathrm{pOB}+\mathrm{OEC}$ cultivated for $14 \mathrm{~d}$ ). Statistical significance was set at $* p<0.05, n=3$. 
A

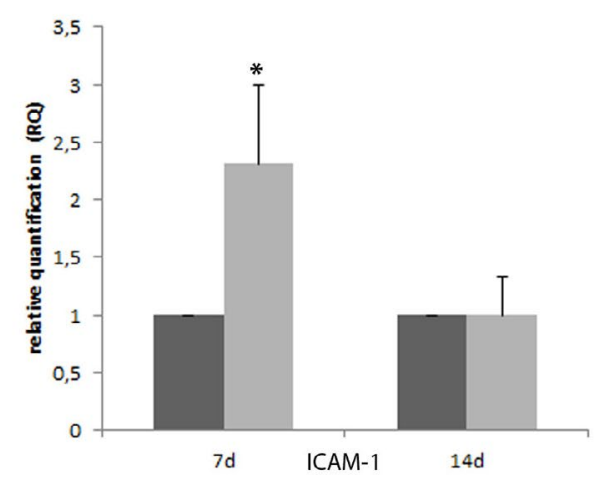

C

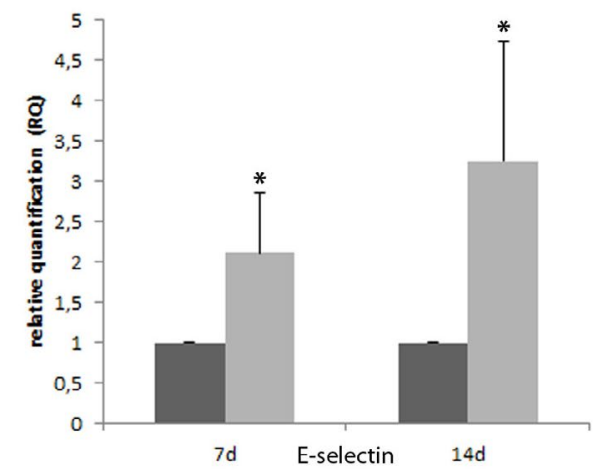

B

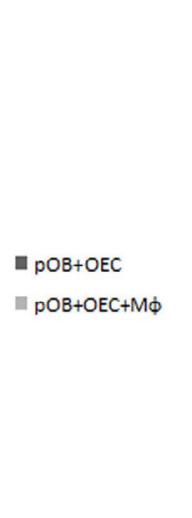

DOB+OEC+M

D

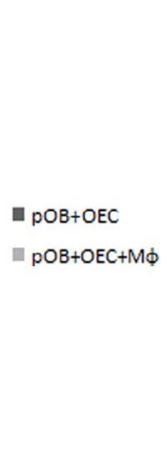

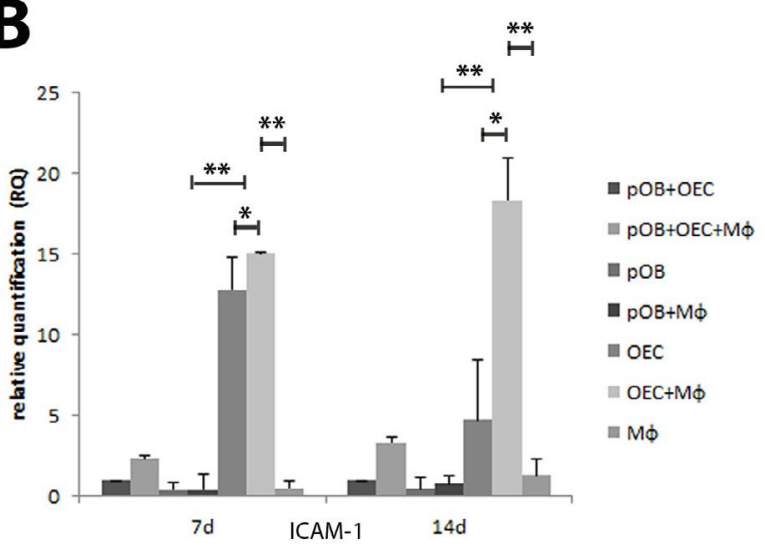

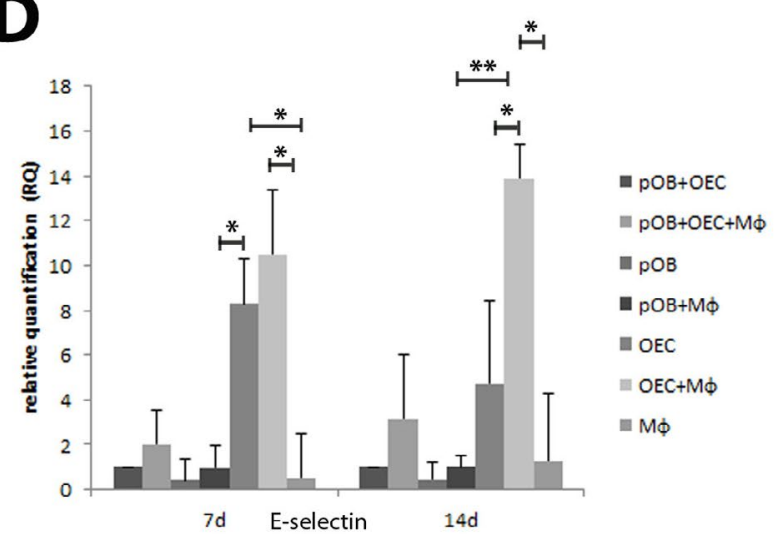

Fig. 4. Effect of macrophage-induced THP-1 on the expression of inflammation-associated adhesion molecules ICAM-1 and E-selectin. (A,C) Treatment of co-cultures with MФ leads to an increase in the expression of ICAM-1 and E-selectin on the mRNA level as evaluated using quantitative real time RT-PCR compared to the co-culture consisting of pOBs and OECs. Upregulation of ICAM-1 after $7 \mathrm{~d}$ of triple-cultivation as well as E-selectin upregulation in response to $7 \mathrm{~d}$ and $14 \mathrm{~d}$ of $\mathrm{M} \Phi$ treatment was found to be statistically significant $\left({ }^{*} p<0.05\right)(n=7)$. (B,D) The expression of ICAM-1 and E-selectin in the appropriate mono-cultures as well as in double co-cultures consisting of OEC+MФ and pOB $+\mathrm{M} \Phi$ revealed a significantly higher expression of ICAM-1 in OECs and OEC+MФ co-cultures after both time points $\left(\mathbf{B}, * p<0.05, n=3\right.$ and $\left.{ }^{* *} p<0.03\right)$. In general, E-selectin expression is also higher in endothelial cell cultures compared to pOB cultures after $14 \mathrm{~d}\left(\mathbf{D},{ }^{*} p<0.05, n=3\right)$.

vessel formation. The expression of VEGF was analysed in triple-cultures of pOBs, OECs and macrophage-induced THP-1 (MФ) at the mRNA level using quantitative real time RT-PCR (qRT-PCR) (Fig. 5A), as well as at the protein level, evaluated using ELISA (Fig. 5C), these data being compared to the double co-cultures (Fig. 5, $n=7$ ). The relative expression of VEGF at the mRNA level is upregulated after 7 and $14 \mathrm{~d}$ of triple-cultivation compared to the control cultures and statistical significance was established for the 14 day time point (Fig. 5A, ${ }^{*} p<0.05$ ). The higher expression of VEGF at the gene expression level in response to THP-1 treatment in co-cultures was additionally confirmed on the protein level. A significantly higher concentration of VEGF in cell culture supernatants of triple-cultures after $14 \mathrm{~d}$ of cultivation was assessed using ELISA (Fig. $5 \mathrm{C},{ }^{* *} p<0.03$ ). After $7 \mathrm{~d}$ of triple- culture, no differences were found in the concentration of VEGF in cell culture supernatants when compared to the double co-culture without MФ (Fig. 5C). To obtain insight into the potential origin of VEGF in the tripleculture system, mono-cultures as well as differently combined double co-cultures $(\mathrm{OEC}+\mathrm{M} \Phi$ and $\mathrm{pOB}+\mathrm{M} \Phi)$ were investigated in terms of VEGF expression at both mRNA and protein levels (Fig. 5B,D, $n=3$ ). On the gene expression level, pOB- and $\mathrm{M} \Phi$-mono-cultures as well as $\mathrm{pOB}+\mathrm{M} \Phi$ co-cultures revealed a quite high expression after 7 and $14 \mathrm{~d}$ of cultivation (Fig. $5 \mathrm{~B}, * p<0.05$ ). This was in accordance with results from ELISA, also indicating VEGF release mainly from pOB- and MФ-mono-cultures as well as from co-cultures consisting of pOBs $+\mathrm{M} \Phi$ (Fig. $5 \mathrm{D}, * p<0.05$ and $* * p<0.03)$, but not from OEC monocultures. 
A

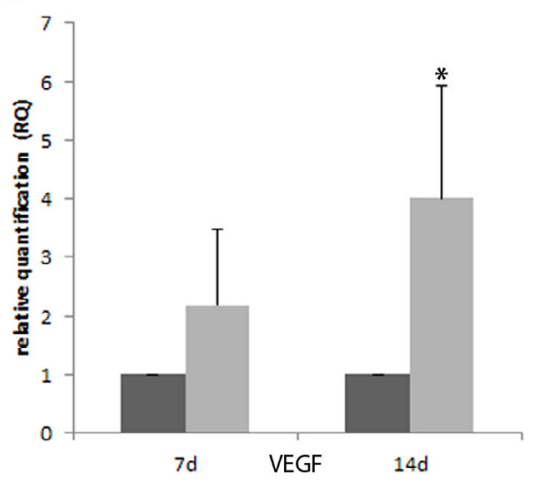

C

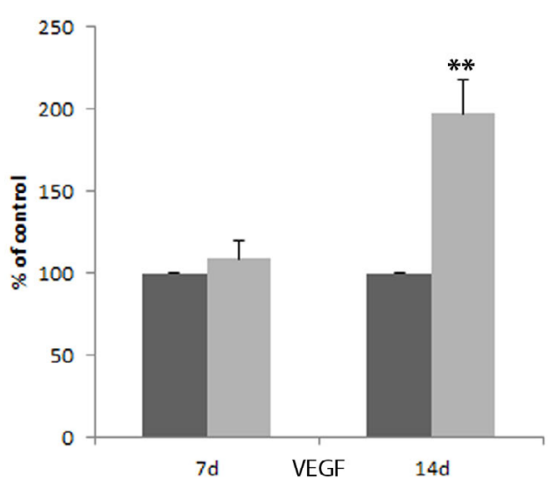

B

DOB+OEC

DOB+OEC+M $\phi$

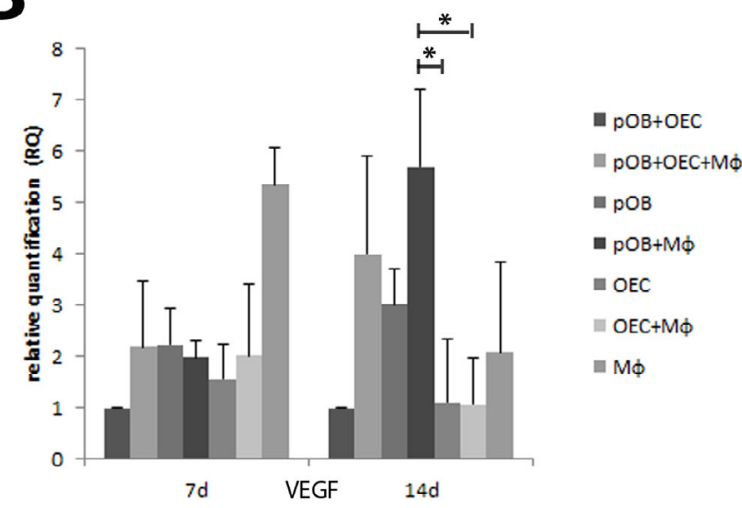

D

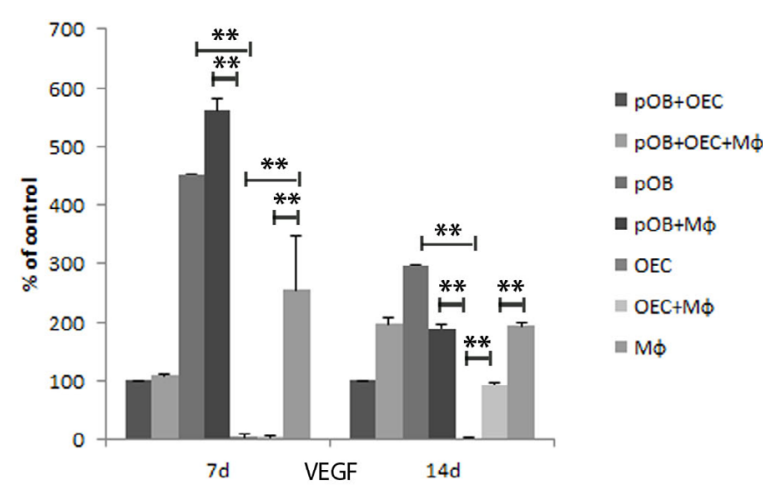

Fig. 5. Effect of macrophage-induced THP-1 on the expression of the proangiogenic growth factor VEGF at the mRNA as well as the protein level. (A) In response to MФ exposure, the expression of VEGF increased at the mRNA level after $7 \mathrm{~d}$ as well as $14 \mathrm{~d}$ of triple-culturing, whereas statistical significance was only achieved for the upregulated expression at the $14 \mathrm{~d}$ time point of triple-culture $\left({ }^{*} p<0.05\right)$. (C) Protein concentration of VEGF in supernatants of $\mathrm{M} \Phi$ treated cultures was also statistically significantly increased after $14 \mathrm{~d}$ of treatment, as evaluated using an enzyme-linked immunosorbent assay (ELISA) $\left(\mathbf{C},{ }^{* *} p<0.03, n=7\right)$. (B,D) Analysing VEGF expression as well as VEGF protein content also in mono-cultures as well as in co-cultures consisting of $\mathrm{OEC}+\mathrm{M} \Phi$ and $\mathrm{pOB}+\mathrm{M} \Phi$ determined the VEGF expression and release mainly by the pOBs and by the M $\Phi$-mono-cultures ${ }^{*} p<0.05$ and $* * p<0.03, n=3)$.

\section{Inhibition of VEGF results in blocking of macrophage-induced angiogenic activation of OECs in the triple-culture}

To confirm that the macrophage-induced increased formation of angiogenic structures in the triple-culture strongly correlates with a higher secretion of VEGF in this system, VEGF blocking experiments were performed (Fig. 6). Therefore, soluble VEGF was blocked in the triple-cultures using conditioned medium from soluble Flk1 (VEGF receptor 2)-producing mesenchymal stem cells (MSCs) for 7 and $14 \mathrm{~d}$. BMSC were retrovirally transduced to express a soluble form of the mouse VEGF receptor-2 (sFlk-1) linked in a bicistronic construct to a truncated version of mouse CD8a, used as FACS-sortable marker. The Flk1 in this model system serves as a VEGF inhibitor. The efficiency of transduction was $87 \%$ for the sFlk1-expressing cells. Supernatants of genetically modified BMSC contained $15.7 \pm 0.8 \mathrm{ng} / \mathrm{mL}$ of mouse sFlk-1 (data not shown). VEGF concentration was assessed in cell culture supernatants using ELISA (Fig. $6 \mathrm{I}, n=3, * p<0.05$ and $* * p<0.03)$. It could be shown that conditioned medium from sFLK1-producing MSCs significantly reduced the VEGF protein content in the triple-culture supernatants in direct comparison of the VEGF concentration in supernatants of the control coand triple-cultures. In fact, total abrogation was achieved, so that VEGF was undetectable in the Flk1-conditioned medium groups. Furthermore, in accordance with the reduction of the VEGF protein amount in triple-cultures treated with sFlk1, the formation of angiogenic structures was clearly blocked in these triple-cultures (Fig. 6C,G). Nearly no formation of microvessel-like structures could be observed when triple-cultures treated with conditioned medium from sFlk1-producing MSCs were evaluated through staining for the endothelial marker CD31 (Fig. $6 \mathrm{C}, \mathrm{G}, n=3$ ). The formation of angiogenic structures in the triple-cultures with VEGF-blocking sFlk1 medium was compared to the control-co-cultures (Fig. 6A,E, $n=3$ ), to 

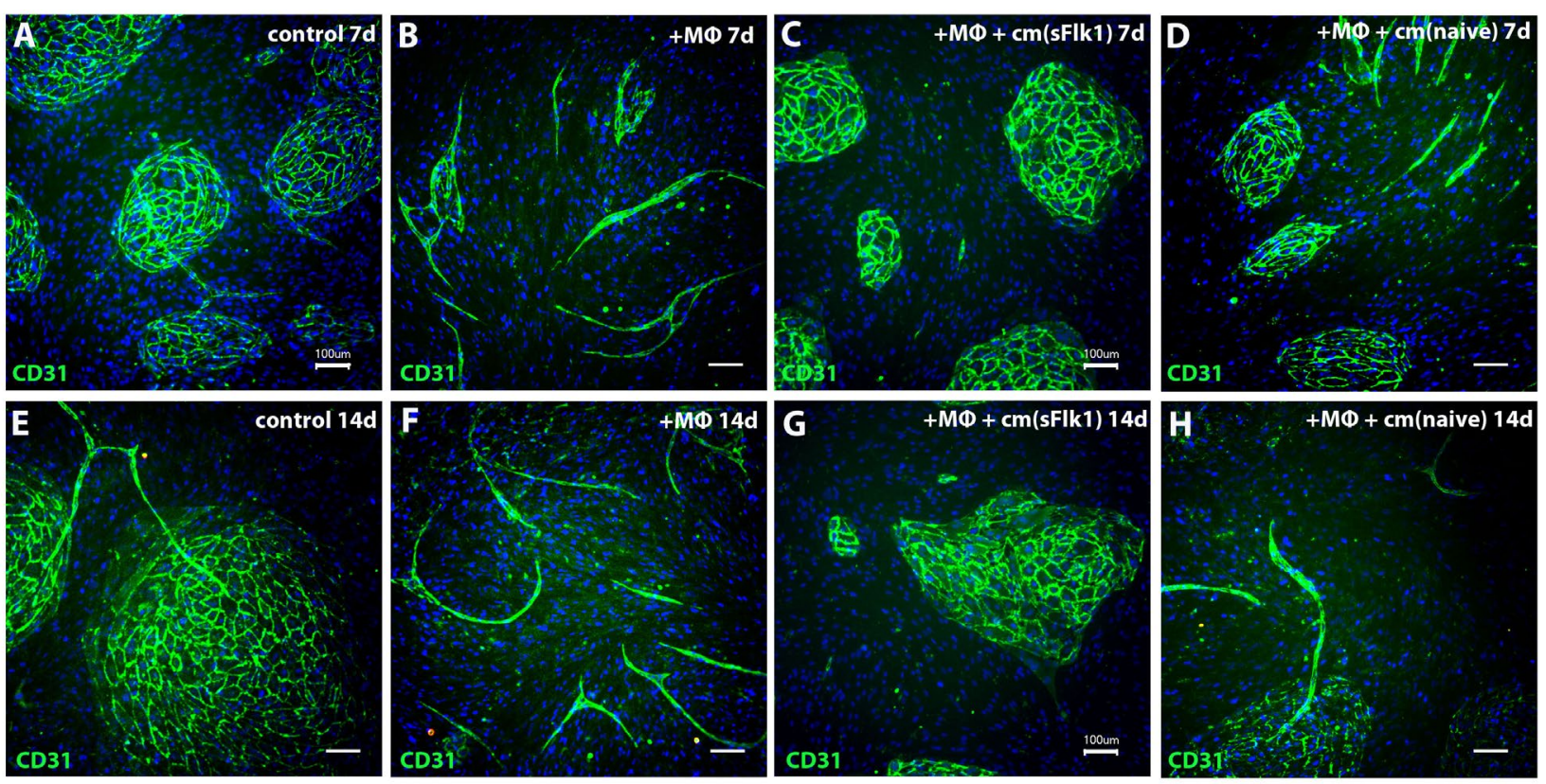

I

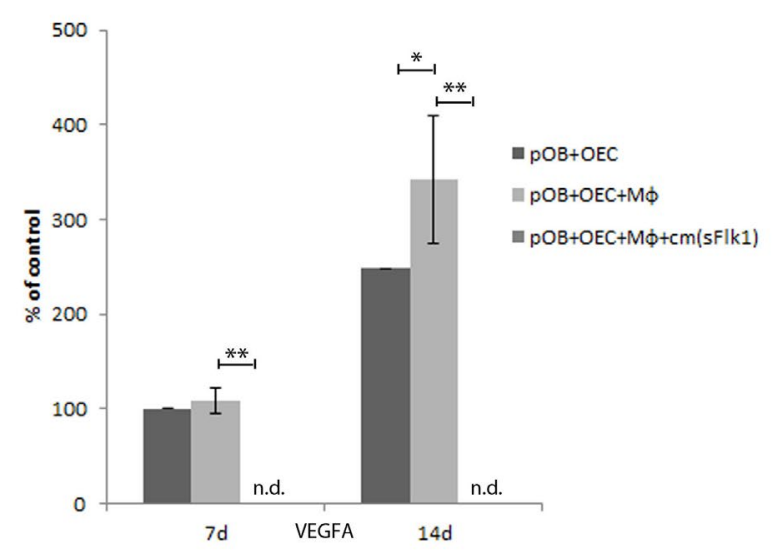

Fig. 6. Inhibition of macrophage-mediated angiogenic activation of OECs in the triple-culture using sFlk1 (A-H) The formation of angiogenic structures was clearly blocked in sFlk1 treated triple-cultures after both cultivation time points as evaluated by staining for the endothelial marker CD31 (C, G, $n=3)$. The formation of angiogenic structures in the triple-cultures with VEGF blocking sFlk1 medium was compared to control co-cultures (A,E, $n=3)$, to control triple-cultures (B,F, $n=3)$, as well as to control triple-cultures with conditioned medium from the naïve $\operatorname{MSCs}(\mathbf{D}, \mathbf{H}, n=3)$. (I) Soluble VEGF was blocked in the triple-cultures using conditioned medium from soluble Flk1 (VEGF receptor 2)-producing MSCs for 7 and $14 \mathrm{~d}$ and VEGF concentration was assessed in cell culture supernatants using $\operatorname{ELISA}(n=3$, ${ }^{*} p<0.05$ and ${ }^{* *} p<0.03 ;$ n.d. $=$ not detectable). the control triple-cultures (Fig. 6B,F, $n=3$ ) as well as to control triple-cultures with conditioned medium from the naïve MSCs (Fig. 6D,H, $n=3$ ).

\section{Effects of THP-1 on the osteogenic differentiation in co-cultures of pOBs and OECs}

For successful bone repair, a tissue engineered bone construct that combines a rapid connection to the vasculature as well as the capability to maintain an osteoblastic phenotype would be advantageous for a therapeutic intervention in bone tissue engineering. Bone is a highly vascularised tissue and bone formation is critically under the control of the interaction between the endothelium and bone cells. Therefore, another point of interest during this study was to analyse the osteogenic potential of the triple-culture system with respect to a maintained osteoblastic differentiation. Alzarin red staining and quantification was used to determine the mineralisation content in co- and triple-cultures after 7 and $14 \mathrm{~d}$ of cultivation. In general, the mineralisation content was slightly higher after $14 \mathrm{~d}$ compared to 7 days of cultivation, as determined in $\mu \mathrm{M}$ Alizarin $/ \mathrm{mg}$ protein (Fig. 7A, $n=3$ ). Nevertheless, there was no statistically significant difference in Alizarin Red concentration between double co-cultures and triple-cultures, neither after $7 \mathrm{~d}$ nor $14 \mathrm{~d}$ of cultivation. In addition, the expression level of several osteogenic differentiation markers was compared in double co-cultures consisting of pOBs and OECs and triple-cultures of pOBs, OECs and MФ (Fig. 7B-D, $n=3$ ). Osteocalcin (Fig. 7B), osteopontin (Fig. 7C) and the osteogenic transcription factor RUNX2 (Fig. 7D) did not differ in their expression in triple-cultures compared to co-cultures after $7 \mathrm{~d}$ of cultivation. However, after $14 \mathrm{~d}$ of cultivation, there seems to be a slight upregulation of these molecules in the $M \Phi$-treated triple-cultures, although statistical significance could only be assessed for the upregulated expression of osteocalcin in the triple-cultures (Fig. 7B, $n=3,{ }^{*} p<0.05$ ).

\section{Discussion}

Over the past decade, different approaches have been developed to provide a rapid connection of tissue engineered bone constructs to the host vasculature to ensure a sufficient supply of oxygen and nutrients needed for the 
A

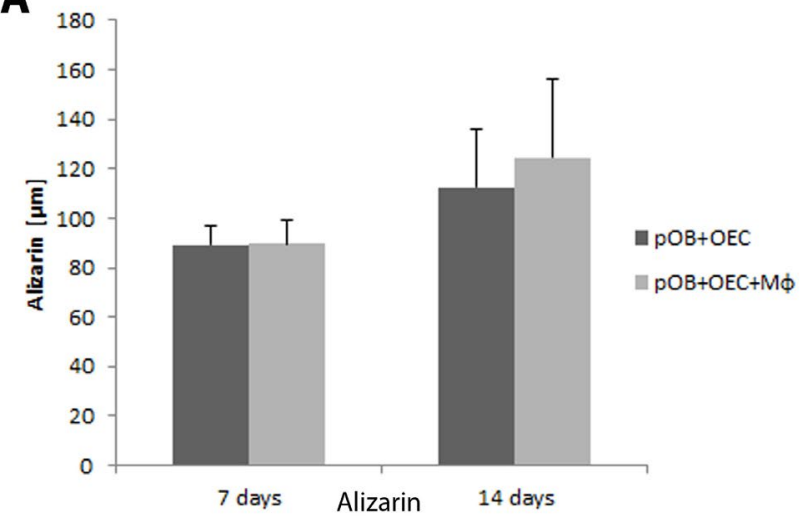

C

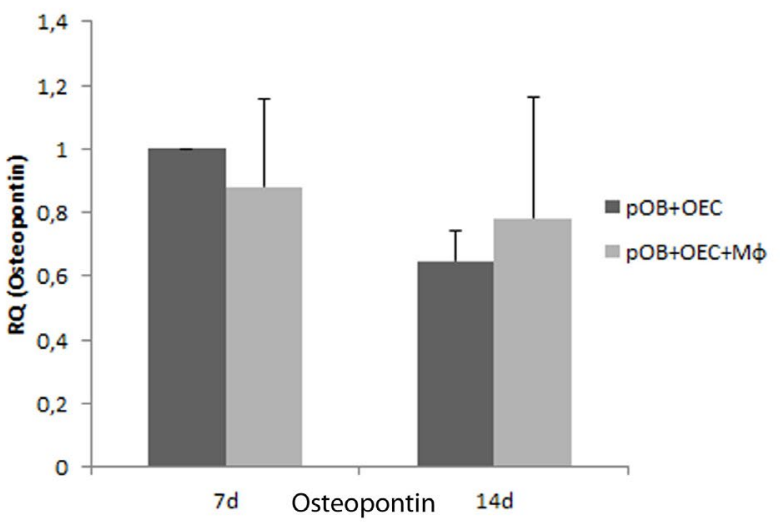

B

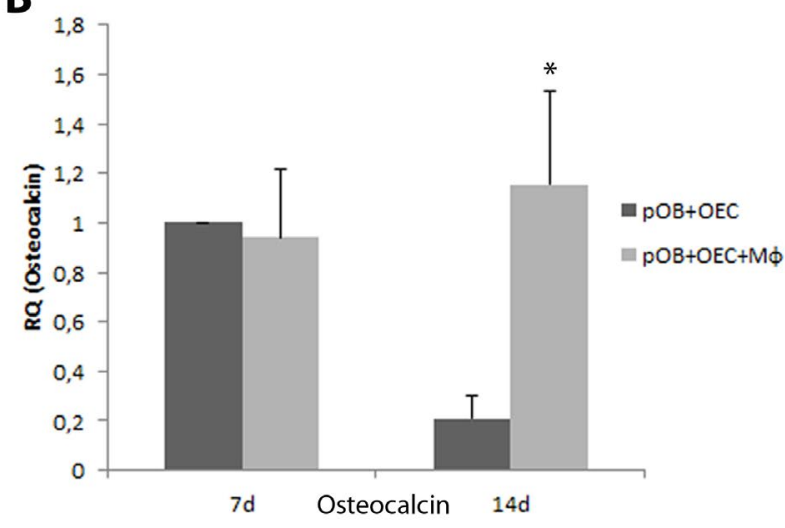

D

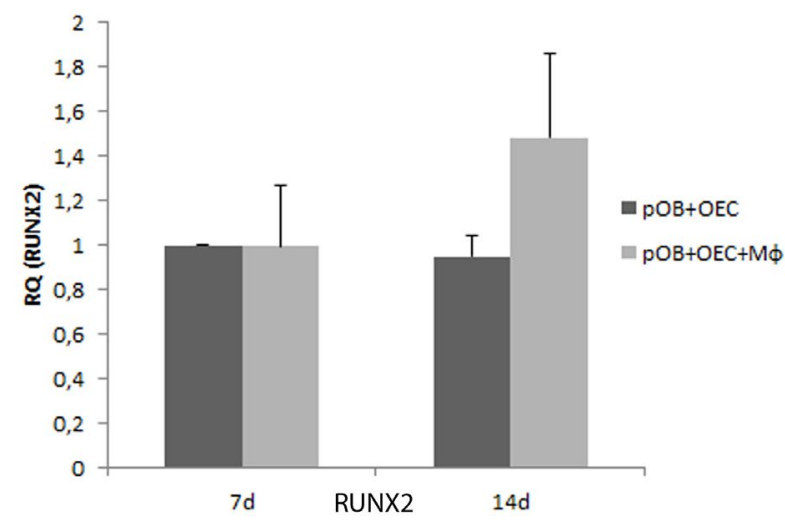

Fig. 7. Osteogenic differentiation in the triple-culture system after 7 and $14 \mathrm{~d}$ of cultivation. (A) Alizarin red staining and quantification was used to determine the mineralisation content in co- and triple-cultures. The mineralisation content was slightly higher after $14 \mathrm{~d}$ of cultivation, determined in $\mu \mathrm{M}$ Alizarin/mg protein $(n=3)$. No difference in Alizarin Red concentration between co-cultures and triple-cultures was detected, neither after $7 \mathrm{~d}$ nor $14 \mathrm{~d}$ of cultivation. (B-D) The expression level of osteocalcin, osteopontin and RUNX2 was compared in co-cultures consisting of pOBs and OECs and triple-cultures of pOBs, OECs and MФ. Osteocalcin (B), osteopontin (C) and RUNX2 (D) did not differ in their expression in triple-cultures compared to co-cultures after $7 \mathrm{~d}$ of cultivation. After $14 \mathrm{~d}$ of cultivation, there seems to be a slight upregulation of these molecules in the МФ-treated triple-cultures. Relative gene expression was determined using the $\Delta \Delta \mathrm{Ct}$ method and gene expression was compared by setting control co-cultures cultivated for $7 \mathrm{~d}$ to 1 . Statistical significance was only achieved for the upregulated expression of osteocalcin in the triple-cultures $(n=3, * p<0.05)$.

survival of the construct. These vascularisation strategies include different approaches, such as the use of delivery systems for proangiogenic growth factors (Geiger et al., 2005; Gu et al., 2004) or the generation of prevascularised tissues by incorporation of endothelial cells or endothelial progenitor cells (Rivron et al., 2008; Rouwkema et al., 2006). Another beneficial approach is the use of complex co-culture systems consisting of different cell types to improve the vascularisation process (Fuchs et al., 2007; Kaigler et al., 2003). In addition, a simple solution to enhance the vascularisation of tissue engineered constructs might be to utilise the natural responses of the human body.

Under physiological conditions, two different ways of inducing the process of angiogenesis exist, namely hypoxia- and inflammation-induced angiogenesis during wound healing. Inflammatory cells such as macrophages $(\mathrm{M} \Phi)$ or monocytes are essential and dominant cells during wound healing and they are known to play a significant role in the in vivo induction of new blood vessel formation via upregulation of different proinflammatory cytokines, including $\mathrm{TNF} \alpha$, and growth factors, such as VEGF. From the literature, it is known that macrophages can exert a spectrum of different functions. Depending on their phenotype they can adopt a proinflammatory (M1macrophage) or an angiogenic role (M2-macrophage) (Lamagna et al., 2006). PMA treatment of the THP-1 cell line revealed an upregulation of IL-1 $\beta$, a well-known M1 marker, as well as the upregulation of TGFß, IGF1 and a higher secretion of the cytokines IL-8 and IL-10, all defined as markers for M2 macrophages, indicating a dual role of the used $\mathrm{M} \Phi$ in the triple-culture system. Nevertheless, the role of macrophages in the regulation of angiogenesis seems to be very complex. Recently, it has been shown that tissue macrophages act as chaperones for vascular anastomosis and stimulate tissue vascularisation (Fantin et al., 2010). In this study, we analysed the different 
modulatory activity that macrophages may have on the established co-culture system consisting of primary human osteoblasts (pOBs) and human outgrowth endothelial cells (OECs) in terms of inflammation-induced angiogenic activation with regard to proinflammatory cytokines, inflammatory adhesion molecules and proangiogenic growth factor expression after a short time cultivation point (7 d) and a longer cultivation period (14 d) with the aim to improve the angiogenic capabilities of the co-culture system.

During wound healing, macrophages play an ubiquitous role. The initial stage of wound healing is triggered by fibrin clot formation (wound matrix) that automatically initiates the inflammatory process (Reinke and Sorg, 2012). The inflammation phase starts when activated platelets from the fibrin clot secrete different growth factors that attract leukocytes, i.e. neutrophils and monocytes (Martin, 1997). Once monocytes are at the site of damage, they differentiate into macrophages and phagocytose pathogens and necrotic tissue before they secrete proinflammatory cytokines, including TNF $\alpha$, IL-1 $\beta$ and IL-6, which amplify the inflammatory response (Eming et al., 2007; Werner and Grose, 2003). In response to treatment of co-cultures with macrophage-induced THP-1 (MФ), the expression of different proinflammatory cytokines, such as IL-6, IL-8 and TNF $\alpha$, was upregulated at the mRNA level after both stimulation time points $(7 \mathrm{~d} / 14 \mathrm{~d})$ in the triple-cultures, whereas IL-6 and IL-8 expression was found to be statistically significantly upregulated only after the longer incubation period ( 2 weeks) with MФ exposure. The human THP-1 cell line was first established thirty years ago by Tsuchiya et al. (1980) and proved to be one of the most used cell lines to analyse monocyte/ macrophage function in vitro. It is already well documented that the release of proinflammatory cytokines in differently treated THP-1 cells is such that they could induce an inflammatory response. Thus, when THP-1 are exposed to LPS (lipopolysaccharide) or insulin, for instance, the concentration of TNF $\alpha$ and IL- $1 \beta$ released from the stimulated THP-1 increases significantly (He et al., 2012; Iida et al., 2001). According to the literature, when THP-1 cells are treated with PMA, the number of CD11b positive cells, which is often used as a pan-macrophage marker, clearly increases (Lu and Pitha, 2001). In addition, the higher expression of CD14 and TLR2 document an ongoing differentiation of the THP-1 into macrophages in response to PMA treatment (Li et al., 2002). Several different studies have been published, examining the interaction between THP-1 cells and endothelial cells in various experimental settings (Li et al., 2006; Zhang et al., 2008). However, there is less known about the inflammatory effect of macrophage induced THP-1 (MФ) in combination with co-culture systems in general. In the present study we have been able to show that the pure combination of $\mathrm{M} \Phi$ with the established co-culture system of pOBs and OECs leads to an upregulation of the proinflammatory cytokines IL- 6 , IL- 8 and TNF $\alpha$ and provides a first indication that this combination alone seems to induce an inflammatory-type response in vitro. Nevertheless, control experiments analysing the gene expression levels of these proinflammatory factors in mono-cultures as well as in differently combined double co-cultures revealed that $\mathrm{M} \Phi$ were not able to potentiate the proinflammatory signal in combination with OECs or pOBs alone. A triple-culture is a highly regulated and complex system and it is difficult to distinguish which cell type is exerting which function. This is especially the case when more than one cell type in the triple-culture system can produce a biologically active molecule. However, the control experiments can be conceived in such a way as to indicate that the different cell types act together in a cooperating way. The in vitro results presented here strongly confirm the need for all 3 cell types to induce an inflammatory response, which appears to reflect the in vivo situation.

In addition, the expression of the adhesion molecules E-selectin and ICAM-1, which are strongly involved in the interaction between activated macrophages and endothelial cells during the process of inflammation were also found to be upregulated on the mRNA level in triple-cultures of pOBs, OECs and M $\Phi$ compared to the mRNA expression of these molecules in the co-cultures without addition of macrophage-induced THP-1 (MФ). This documents an ongoing proinflammatory stimulus in the co-culture when combined with $M \Phi$. During inflammation, the first interaction between macrophages and endothelial cells is mainly mediated through E-selectin and P-selectin which are expressed on endothelial cells and integrins expressed on macrophages (leukocyte rolling) (Abbassi et al., 1993; Dore et al., 1993; Gaboury and Kubes, 1994). Macrophageendothelial cell adhesion at a stronger level is mediated through ICAM-1 and vascular cell adhesion molecule-1 (VCAM-1), before macrophages transmigrate and initiate the proliferative phase of wound healing which finally leads to new blood vessel formation (Fernandez-Borja et al., 2010; Libby et al., 2002). As expected, ICAM-1 and E-selectin are strongly expressed in OEC mono-cultures compared with pOB mono-cultures (Rahman et al., 1998; Wong and Dorovini-Zis, 1992) and the expression levels could be significantly increased in OECs by adding M $\Phi$ in control double co-culture experiments. It is already known that ICAM-1 and E-selectin are expressed on the surface of the vascular endothelium and can be induced to high gene expression levels in response to various inflammatory mediators or inflammatory cytokines, such as IL-6, IL-8 or TNFa (Antonelli et al., 2001; Springer, 1994; van de Stolpe and van der Saag, 1996; Wyble et al., 1997).

Subsequently, in the proliferative phase of inflammation and subsequent wound repair, the process of angiogenesis to restore the vascular network in the wound surface is initiated. The inflammation-induced formation of new capillaries proceeds through a series of steps and is under the control of different growth factors. Activated leukocytes secrete PDGF and TGF $\beta$ that attract fibroblasts to the wound, where they proliferate and produce extracellular matrix in the granulation tissue (Eming et al., 2007). Both macrophages as well as fibroblasts within the wound region secrete the proangiogenic growth factor VEGF (Gurtner et al., 2008; Werner and Grose, 2003). VEGF production, in turn, leads to an increase in vessel permeability, endothelial migration and finally to new blood vessel formation. Treatment of co-cultures of pOBs and OECs 
with macrophage-induced THP-1 (MФ) for two different time periods ( $7 \mathrm{~d}$ and $14 \mathrm{~d}$ ) resulted in an increase in the number of microvessel-like structures after both triplecultivation time points, compared to the control double co-cultures. However, treating co-cultures consisting of pOBs and OECs with conditioned medium derived from macrophage supernatants did not lead to the formation of angiogenic structures in this system in control experiments, documenting the essential need for the direct contact of the 3 cell types in this system (Kofler et al., 2011; Nucera et al., 2011). The higher number of angiogenic structures in the direct triple-cultures correlated with a significantly higher concentration of the proangiogenic growth factor VEGF in cell culture supernatants of triple-cultures, as determined using ELISA. In addition, VEGF mRNA was upregulated in the co-cultures in response to $\mathrm{M} \Phi$ treatment. It has already been shown by Tjiu et al . (2009), that THP-1 derived macrophages are able to induce the formation of angiogenic structures via VEGF and bFGF upregulation in different settings. In fact, direct cell contact of activated macrophages, pOBs and OECs seems to play an important role in the regulation of angiogenesis by increasing the production and secretion of different growth factors and cytokines, amongst others VEGF (Ferrara, 2000; McLaren et al., 1996), IL-6, IL-8 and TNF $\alpha$ (Ono et al., 1999; Sunderkotter et al., 1994), which could also be confirmed in the present study. VEGF blocking experiments using conditioned medium obtained from Flk1 expressing cells was added to the triple-culture and analysed in terms of vessel-formation. Compared to the control (triple-culture) the formation of angiogenic structures could be blocked using soluble Flk1, thus clearly documenting an essential role for the VEGF-induced formation of angiogenic structures in the triple-culture system.

Furthermore, it could be shown that the triple-culture seems to have at least no negative effect on osteogenic differentiation in this system, as evaluated using gene expression studies for the osteogenic markers osteocalcin, osteopontin and RUNX2 as well as by analysing the calcification level in the triple-culture system through Alizarin Red quantification. A positive effect on the osteogenic differentiation of osteoblasts in co-culture with macrophages/monocytes was already documented in a number of recent studies (Guihard et al., 2012; Pirraco et al., 2013). Collectively with results from previous studies, the present data document the VEGF-mediated up-regulation of pro-angiogenic reactions in a novel triple-culture system consisting of human pOBs, OECs and macrophages. Our data suggest the possibility of actively using pro-inflammatory signals to accelerate vascularisation in bone tissue engineering applications.

\section{Conclusion}

On the basis of an in vitro co-culture model to delineate different mechanisms during the vascularisation process in bone repair, this study was undertaken to test the hypothesis that new vessel formation can be influenced in the co-culture model by cell-related pro-inflammatory factors in a manner simulating the in vivo situation. The current findings demonstrate that mimicking the natural cell response using the macrophage-induced THP-1 cell line as inflammatory stimulus in the established co-culture of pOBs and OECs leads to an angiogenic activation of OECs, as seen by an increase of the formation of microvessel-like structures in the triple-culture system. This acceleration of angiogenic structure formation in response to the presence of $\mathrm{M} \Phi$ correlated with a higher secretion of the proangiogenic factor VEGF, this being further validated by VEGF blocking experiments. The higher secretion of VEGF might be at least partly initiated through an upregulation of proinflammatory cytokines and adhesion molecules induced by the addition of macrophages, indicating an ongoing inflammatory-like process in the triple-culture consisting of pOBs, OECs and macrophage-induced THP-1. Importantly, the tripleculture was shown to exert no negative effect on osteogenic differentiation in this system. Nevertheless, the present study has to be considered as fundamental research, and so additional studies are needed to analyse the underlying mechanisms and the basic control machinery during the bone repair process with regard to inflammation-induced angiogenesis. However, the data provide evidence that the complexity of a double-and triple-culture system with relevant human cells mirrors many of the biologically important, coordinated steps in wound healing in vivo. Of further interest to the tissue engineering field are future studies on suitable scaffolds to determine whether this macrophage-mediated pro-angiogenic effect can occur in the triple-culture system in three-dimensional biomaterials.

\section{Acknowledgements}

The authors would like to thank B. Pavic and A. Sartoris for their excellent technical assistance and C. J. Kuo (Stanford University) for kindly providing the sFlk1-Fc cDNA This work was financially supported by grants from the University Medical Centre Mainz (MAIFOR 9728901) and BMBF for the German-Chinese Young Investigator Group (grant number 0315033) and the European Union FP7 grant Disc Regeneration (CP-IP 213904). We wish to confirm that there are no known conflicts of interest associated with this publication and there has been no significant financial support for this work that could have influenced its outcome.

\section{References}

Abbassi O, Kishimoto TK, McIntire LV, Anderson DC, Smith CW (1993) E-selectin supports neutrophil rolling in vitro under conditions of flow. J Clin Invest 92: 2719-2730.

Adams RH, Alitalo K (2007) Molecular regulation of angiogenesis and lymphangiogenesis. Nat Rev Mol Cell Biol 8: 464-478.

Antonelli A, Bianchi M, Crinelli R, Gentilini L, Magnani M (2001) Modulation of ICAM-1 expression in ECV304 cells by macrophage-released cytokines. Blood Cells Mol Dis 27: 978-991. 
Diegelmann RF, Evans MC (2004) Wound healing: an overview of acute, fibrotic and delayed healing. Front Biosci 9: 283-289.

Dohle E, Fuchs S, Kolbe M, Hofmann A, Schmidt H, Kirkpatrick CJ (2010) Sonic hedgehog promotes angiogenesis and osteogenesis in a coculture system consisting of primary osteoblasts and outgrowth endothelial cells. Tissue Eng Part A 16: 1235-1237.

Dohle E, Fuchs S, Kolbe M, Hofmann A, Schmidt H, Kirkpatrick CJ (2011) Comparative study assessing effects of sonic hedgehog and VEGF in a human co-culture model for bone vascularisation strategies. Eur Cell Mater 21: 144-156.

Dore M, Korthuis RJ, Granger DN, Entman ML, Smith CW (1993) P-selectin mediates spontaneous leukocyte rolling in vivo. Blood 82: 1308-1316.

Eming SA, Krieg T, Davidson JM (2007) Inflammation in wound repair: molecular and cellular mechanisms. J Invest Dermatol 127: 514-525.

Fantin A, Vieira JM, Gestri G, Denti L, Schwarz Q, Prykhozhij S, Peri F, Wilson SW, Ruhrberg C (2010) Tissue macrophages act as cellular chaperones for vascular anastomosis downstream of VEGF-mediated endothelial tip cell induction. Blood 116: 829-840.

Fernandez-Borja M, van Buul JD, Hordijk PL (2010) The regulation of leucocyte transendothelial migration by endothelial signalling events. Cardiovasc Res 86: 202-210.

Ferrara N (2000) Vascular endothelial growth factor and the regulation of angiogenesis. Recent Prog Horm Res 55: 15-35; discussion 35-16.

Finkenzeller G, Arabatzis G, Geyer M, Wenger A, Bannasch H, Stark GB (2006) Gene expression profiling reveals platelet-derived growth factor receptor alpha as a target of cell contact-dependent gene regulation in an endothelial cell-osteoblast co-culture model. Tissue Eng 12: 2889-2903.

Fuchs S, Hermanns MI, Kirkpatrick CJ (2006) Retention of a differentiated endothelial phenotype by outgrowth endothelial cells isolated from human peripheral blood and expanded in long-term cultures. Cell Tissue Res 326: 79-92.

Fuchs S, Hofmann A, Kirkpatrick CJ (2007) Microvessel-like structures from outgrowth endothelial cells from human peripheral blood in 2-dimensional and 3-dimensional co-cultures with osteoblastic lineage cells. Tissue Eng 13: 2577-2588.

Fuchs S, Jiang X, Schmidt H, Dohle E, Ghanaati S, Orth C, Hofmann A, Motta A, Migliaresi C, Kirkpatrick CJ (2009) Dynamic processes involved in the pre-vascularization of silk fibroin constructs for bone regeneration using outgrowth endothelial cells. Biomaterials 30: 1329-1338.

Gaboury JP, Kubes P (1994) Reductions in physiologic shear rates lead to CD11/CD18-dependent, selectinindependent leukocyte rolling in vivo. Blood 83: 345-350.

Geiger F, Bertram H, Berger I, Lorenz H, Wall O, Eckhardt C, Simank HG, Richter W (2005) Vascular endothelial growth factor gene-activated matrix (VEGF165GAM) enhances osteogenesis and angiogenesis in large segmental bone defects. J Bone Miner Res 20: 2028-2035.

Grellier M, Granja PL, Fricain JC, Bidarra SJ, Renard M, Bareille R, Bourget C, Amedee J, Barbosa
MA (2009) The effect of the co-immobilization of human osteoprogenitors and endothelial cells within alginate microspheres on mineralization in a bone defect. Biomaterials 30: 3271-3278.

Gu F, Amsden B, Neufeld R (2004) Sustained delivery of vascular endothelial growth factor with alginate beads. J Control Release 96: 463-472.

Guihard P, Danger Y, Brounais B, David E, Brion R, Delecrin J, Richards CD, Chevalier S, Redini F, Heymann D, Gascan H, Blanchard F (2012) Induction of osteogenesis in mesenchymal stem cells by activated monocytes/ macrophages depends on oncostatin $\mathrm{M}$ signaling. Stem Cells 30: 762-772.

Gurtner GC, Werner S, Barrandon Y, Longaker MT (2008) Wound repair and regeneration. Nature 453: 314321.

He X, Shu J, Xu L, Lu C, Lu A (2012) Inhibitory effect of Astragalus polysaccharides on lipopolysaccharideinduced TNF- $\alpha$ and IL- $1 \beta$ production in THP- 1 cells. Molecules 17: 3155-3164.

Helmrich U, Marsano A, Melly L, Wolff T, Christ L, Heberer M, Scherberich A, Martin I, Banfi A (2012) Generation of human adult mesenchymal stromal/stem cells expressing defined xenogenic vascular endothelial growth factor levels by optimized transduction and flow cytometry purification. Tissue Eng Part C Methods 18: 283-292.

Hofmann A, Konrad L, Gotzen L, Printz H, Ramaswamy A, Hofmann C (2003) Bioengineered human bone tissue using autogenous osteoblasts cultured on different biomatrices. J Biomed Mater Res A 67: 191-199.

Horn P, Bork S, Diehlmann A, Walenda T, Eckstein V, Ho AD, Wagner W (2008) Isolation of human mesenchymal stromal cells is more efficient by red blood cell lysis. Cytotherapy 10: 676-685.

Iida KT, Shimano H, Kawakami Y, Sone H, Toyoshima H, Suzuki S, Asano T, Okuda Y, Yamada N (2001) Insulin up-regulates tumor necrosis factor-alpha production in macrophages through an extracellular-regulated kinasedependent pathway. J Biol Chem 276: 32531-32537.

Kaigler D, Krebsbach PH, Polverini PJ, Mooney DJ (2003) Role of vascular endothelial growth factor in bone marrow stromal cell modulation of endothelial cells. Tissue Eng 9: 95-103.

Kofler NM, Shawber CJ, Kangsamaksin T, Reed HO, Galatioto J, Kitajewski J (2011) Notch signaling in developmental and tumor angiogenesis. Genes Cancer 2: 1106-1116.

Kuhnert F, Tam BY, Sennino B, Gray JT, Yuan J, Jocson A, Nayak NR, Mulligan RC, McDonald DM, Kuo CJ (2008) Soluble receptor-mediated selective inhibition of VEGFR and PDGFRbeta signaling during physiologic and tumor angiogenesis. Proc Natl Acad Sci USA 105: 10185-10190.

Lamagna C, Aurrand-Lions M, Imhof BA (2006) Dual role of macrophages in tumor growth and angiogenesis. $\mathrm{J}$ Leukoc Biol 80: 705-713.

Li C, Wang Y, Gao L, Zhang J, Shao J, Wang S, Feng W, Wang X, Li M, Chang Z (2002) Expression of toll-like receptors 2 and 4 and CD14 during differentiation of HL60 cells induced by phorbol 12-myristate 13 -acetate and 
1 alpha, 25-dihydroxy-vitamin D(3). Cell Growth Differ 13: 27-38.

Li R, Mouillesseaux KP, Montoya D, Cruz D, Gharavi N, Dun M, Koroniak L, Berliner JA (2006) Identification of prostaglandin $\mathrm{E} 2$ receptor subtype 2 as a receptor activated by OxPAPC. Circ Res 98: 642-650.

Libby P, Ridker PM, Maseri A (2002) Inflammation and atherosclerosis. Circulation 105: 1135-1143.

Lu R, Pitha PM (2001) Monocyte differentiation to macrophage requires interferon regulatory factor 7. J Biol Chem 276: 45491-45496.

Martin P (1997) Wound healing - aiming for perfect skin regeneration. Science 276: 75-81.

Mayer H, Bertram H, Lindenmaier W, Korff T, Weber H, Weich H (2005) Vascular endothelial growth factor (VEGF-A) expression in human mesenchymal stem cells: autocrine and paracrine role on osteoblastic and endothelial differentiation. J Cell Biochem 95: 827-839.

McLaren J, Prentice A, Charnock-Jones DS, Millican SA, Muller KH, Sharkey AM, Smith SK (1996) Vascular endothelial growth factor is produced by peritoneal fluid macrophages in endometriosis and is regulated by ovarian steroids. J Clin Invest 98: 482-489.

Nucera S, Biziato D, De Palma M (2011) The interplay between macrophages and angiogenesis in development, tissue injury and regeneration. Int J Dev Biol 55: 495-503.

Ono M, Torisu H, Fukushi J, Nishie A, Kuwano M (1999) Biological implications of macrophage infiltration in human tumor angiogenesis. Cancer Chemother Pharmacol 43 Suppl: S69-71.

Pirraco RP, Reis RL, Marques AP (2013) Effect of monocytes/macrophages on the early osteogenic differentiation of hBMSCs. J Tissue Eng Regen Med 7: 392-400.

Rahman A, Kefer J, Bando M, Niles WD, Malik AB (1998) E-selectin expression in human endothelial cells by TNF-alpha-induced oxidant generation and NF-kappaB activation. Am J Physiol 275: L533-544.

Reinke JM, Sorg H (2012) Wound repair and regeneration. Eur Surg Res 49: 35-43.

Rivron NC, Liu JJ, Rouwkema J, de Boer J, van Blitterswijk CA (2008) Engineering vascularised tissues in vitro. Eur Cell Mater 15: 27-40.

Rouwkema J, de Boer J, van Blitterswijk CA (2006) Endothelial cells assemble into a 3-dimensional prevascular network in a bone tissue engineering construct. Tissue Eng 12: 2685-2693.

Springer TA (1994) Traffic signals for lymphocyte recirculation and leukocyte emigration: the multistep paradigm. Cell 76: 301-314.

Sternberg SR (1983) Biomedical image-processing. Computer 16: 22-34.

Sundberg C, Kowanetz M, Brown LF, Detmar M, Dvorak HF (2002) Stable expression of angiopoietin-1 and other markers by cultured pericytes: phenotypic similarities to a subpopulation of cells in maturing vessels during later stages of angiogenesis in vivo. Lab Invest 82: 387-401.

Sunderkotter C, Steinbrink K, Goebeler M, Bhardwaj R, Sorg C (1994) Macrophages and angiogenesis. J Leukoc Biol 55: 410-422.
Tjiu JW, Chen JS, Shun CT, Lin SJ, Liao YH, Chu CY, Tsai TF, Chiu HC, Dai YS, Inoue H, Yang PC, Kuo ML, Jee SH (2009) Tumor-associated macrophage-induced invasion and angiogenesis of human basal cell carcinoma cells by cyclooxygenase-2 induction. J Invest Dermatol 129: 1016-1025.

Tsaryk R, Peters K, Unger RE, Scharnweber D, Kirkpatrick CJ (2007) The effects of metal implants on inflammatory and healing processes. Int J Mater Res 98: 622-629.

Tsuchiya S, Yamabe M, Yamaguchi Y, Kobayashi Y, Konno T, Tada K (1980) Establishment and characterization of a human acute monocytic leukemia cell line (THP-1). Int J Cancer 26: 171-176.

van de Stolpe A, van der Saag PT (1996) Intercellular adhesion molecule-1. J Mol Med 74: 13-33.

Werner S, Grose R (2003) Regulation of wound healing by growth factors and cytokines. Physiol Rev 83: 835-870.

Wong D, Dorovini-Zis K (1992) Upregulation of intercellular adhesion molecule-1 (ICAM-1) expression in primary cultures of human brain microvessel endothelial cells by cytokines and lipopolysaccharide. J Neuroimmunol 39: 11-21.

Wyble CW, Hynes KL, Kuchibhotla J, Marcus BC, Hallahan D, Gewertz BL (1997) TNF-alpha and IL-1 upregulate membrane-bound and soluble E-selectin through a common pathway. J Surg Res 73: 107-112.

Zhang X, Qi R, Xian X, Yang F, Blackstein M, Deng X, Fan J, Ross C, Karasinska J, Hayden MR, Liu G (2008) Spontaneous atherosclerosis in aged lipoprotein lipasedeficient mice with severe hypertriglyceridemia on a normal chow diet. Circ Res 102: 250-256.

Zisch AH, Lutolf MP, Ehrbar M, Raeber GP, Rizzi SC, Davies N, Schmokel H, Bezuidenhout D, Djonov V, Zilla P, Hubbell JA (2003) Cell-demanded release of VEGF from synthetic, biointeractive cell ingrowth matrices for vascularized tissue growth. FASEB J 17: 2260-2262.

\section{Discussion with Reviewers}

Reviewer I: Please provide more information on how the chronology for addition of the different cell types to the cultures was determined.

Authors: The seeding order of OECs and pOBs as well as the culture conditions in the co-culture used in this study are based on the work of Fuchs et al. (2007). In this study the authors analysed effects of the seeding order on the coculture system by seeding both cell types simultaneously or in different seeding steps. Initial formation of microvessellike structures could be observed when OECs were added in a second step. It has already been shown that in this system the pOBs provide, for example, matrix for the OECs to adhere to.

Reviewer I: The authors focussed on macrophage type generally and examined pan-macrophage markers. Have the authors an opinion on the type of macrophages being generated and how the presence of the OECs and OBs might affect this process? 
Authors: It is already known from the literature that macrophages are able to exert a spectrum of different functions. Depending on their phenotype they can adopt a proinflammatory (M1-macrophage) or an angiogenic role (M2-macrophage). In summary, THP-1 cells that were used in this study were pre-treated with PMA, which resulted in an upregulation of IL-1 $\beta$, a well-known M1 marker as well as the upregulation of TGFß, IGF1 and a higher secretion of the cytokines IL-8 and IL-10, all defined as markers for M2 macrophages, indicating a dual role of the used MФ in the triple-culture system during this study. In the scope of this investigation, we did not examine how the OECs or pOBs might affect this process, but are convinced that there could be a modulating role for OECs and pOBs on the type of macrophages. This is currently under investigation.

Reviewer I: Although the study primarily focusses on vasculogenesis the authors do mention that the system is focussed on bone repair. In this context, did the authors examine any osteogenic markers in order to determine whether the presence of the macrophages and subsequent increase in angiogenic factors might have altered osteogenesis by the osteoblasts in the cultures?

Authors: Apart from investigation of the angiogenic phenotype of OECs in response to macrophage treatment, additional studies analysing the osteogenic differentiation in the triple-culture system were performed (Fig. 7). The mineralisation state in co- as well as in triple-cultures was analysed after 7 and $14 \mathrm{~d}$ using Alizarin red quantification and we concluded that there appears to be at least no negative effect in terms of osteogenic mineralisation when macrophages were added to the co-culture. In addition, the expression of the osteogenic differentiation markers osteocalcin and osteopontin and the transcription factor RUNX2 were analysed. After $7 \mathrm{~d}$ of culturing, no difference in the expression of these markers was detectable in response to macrophage treatment, whereas there appeared to be a slight difference in the expression of the osteogenic markers after $14 \mathrm{~d}$ of macrophage treatment compared to the co-culture.

Reviewer II: The present study aimed to investigate intercellular interactions and identify possible factors that could in the future help for tissue engineering applications. The triple-co-culture model used is fascinating and provides very interesting data. However, how do the authors foresee the possible translation to these findings to the clinic? OEC seem to require extended in vitro phases, and primary osteoblasts are not always available and likewise require long time of in vitro cell expansion. Do the authors envisage performing the same type of studies using stem cells (MSC) and other endothelial (progenitor) cell sources (e.g. bone marrow derived, micro-vascular fraction)?

Authors: A co- or a triple-culture is a complex system which is indeed similar to the in vivo situation. The different cell types act together as a unique system and each cell type is influencing the other through paracrine or direct communication, including complicated feedback mechanisms. Therefore, the triple-culture system consisting of pOBs, OECs and macrophages represents a beneficial tool to mimic the natural conditions of the human body, enabling one to gain insight into inter- and intracellular mechanisms through the process of inflammation-induced angiogenesis. Although the isolation and expansion of OECs is time consuming, these cells display a lot of advantages from a therapeutic point of view. Besides their proangiogenic properties in vitro as well as in vivo, these cells can be very easily collected from the peripheral blood with a minimally invasive intervention in comparison with the isolation of stem cells from the bone marrow. Therefore, these cells might serve as a potential autologous cell source for a future therapeutic application. During this study, it has been shown that the pre-cultivation of OECs together with primary osteoblasts and macrophages results in an improvement of the formation of microvessel-like structures in vitro. In addition, the osteogenic capacity seems to be maintained. In the future, this system might be used to generate complex and prevascularised engineered in vitro constructs that might be further used for tissue engineering applications in the clinic. For this, as the reviewer suggests, we could very well envisage using MSC, which should then be driven along the osteogenic lineage, together with another autologous endothelial source. Concerning macrophages in a translational setting, it might be more practicable to use known products of macrophages rather than the cells themselves. Our data certainly point to the fact that pro-inflammatory signals from macrophages could be an early stimulus to promote new vessel growth in the co-culture scenario. 